\title{
A LEP Summary
}

\section{Wilbur Venus* RAL \& CERN}

E-mail: Wilibur. Venus@cern.ch'

ABstRACT: LEP's physics highlights are briefly reviewed in their historical context

\section{INTRODUCTION}

I was asked "to review all of the important results produced by LEP during its 12 years of activity and show what was the situation in various subjects before and after LEP", i.e. to try to put 12 years of feverish activity and well over 1000 papers, and still counting fast in both cases (see Fig. $\left.\underline{1}_{1}^{1}\right)$, into their historical context: in 50 minutes!

After outlining LEP's historical context, I will try to summarise LEP's physics achievements in each category in that context, and then to summarise more globally.

\section{HISTORICAL CONTEXT}

LEP was conceived in 1976, inspired by the success of SPEAR in discovering the $\mathrm{J} / \psi$. This had followed soon after the discoveries of point-like $\nu N$ and high- $Q^{2}$ $e N$ scattering and weak Neutral Currents, and charm was then required theoretically to support the GIM mechanism for suppressing strangeness-changing Neutral Figure 1: LEP publications per year per physics Currents.

\section{LEP publications}

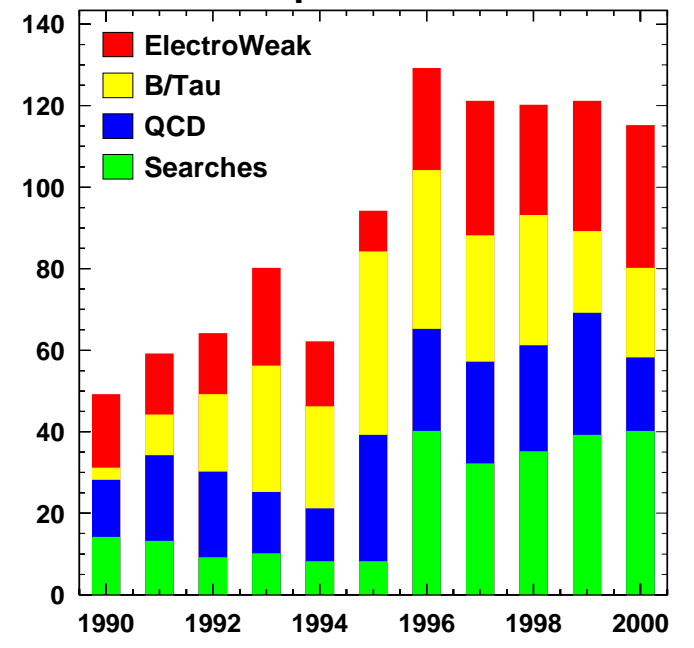

category 1990-2000

Most of the key elements of the revolution of the first half of the 70's, like quarks, gluons, the W, the Z, the Higgs mechanism, and Quantum Field Theory (QFT), had been around for a long time, but were largely ignored. Almost everyone was doing hadron physics (the sixties had seen the great hadron population explosion), and Strong Interaction theorists were into S-matrix theory, dispersion relations, bootstrap models, hadron democracy,

${ }^{*}$ Speaker. 
multiperipheral models, Regge theory, the Veneziano model, etc., and had no time for QFT, which seemed utterly irrelevant to strong coupling and was supposedly withering on the vine. Quarks were widely regarded as epiphenomena rather than as the underlying reality. All that changed with the $\mathrm{J} / \psi$ discovery in 1974 !

The first mention of LEP (but not yet by name) was in a note by Burt Richter [i] The note investigated a LEP-like machine (circumference $\sim 43 \mathrm{~km}$, energy $2 \times 100 \mathrm{GeV}$, luminosity $10^{32}, 8$ interaction points) and found it probably feasible, noted that 'PETRA \& $P E P$ are primarily being built for the contributions they can make to our understanding of hadrons and $Q E D$ ', that Weak Interactions had been studied until then only with neutrino beams with $\mathrm{E}_{\mathrm{cms}}$ of at best $10-20 \mathrm{GeV}$, and that studying Weak Interactions properly really needed $\mathrm{E}_{\mathrm{cms}} \sim 200 \mathrm{GeV}$ or more. This was because by then the point-like "Fermi" cross-section would start to approach the unitarity limit, so something new had to happen: maybe Weinberg-Salam's Z ${ }^{0}$, maybe something else, but surely SOMETHING NEW!

LEP design and physics studies began immediately after that note appeared. The idea was endorsed by the first physics studies report [2]. Design studies soon resulted in a machine that was considered buildable [i]i]. The 1979 Les Houches Summer Study [4] considered the physics case in more detail. This was built on detailed study of $\mathrm{Z}$ production and decay, detailed study of WW production (to check the gauge theory cancellations), and the Higgs search (all unique to LEP); and on searches for new leptons and quarks ("supercharm") and studies of $3 / 4 / .$. jet structures, scaling violations, etc in hadronic events, extending the expected PETRA/PEP factor $\sim 5$ in $\mathrm{E}_{\mathrm{cm}}$ by another factor $\sim 5$.

Glashow's talk at Les Houches is particularly interesting. He said that 'Since the low energy limit is so well confirmed, few can doubt the truth of the (now 'Standard') model' but considered 4 possible scenarios: (1) the 17 parameters scenario, ie the Standard Model (SM), of which he said: "it would be both arrogant and unhistorical to believe that our naive extrapolation from physics at 2 GeV to physics at $200 \mathrm{GeV}$ is likely to be correct in detail", it was "merely the simplest of many possibilities",

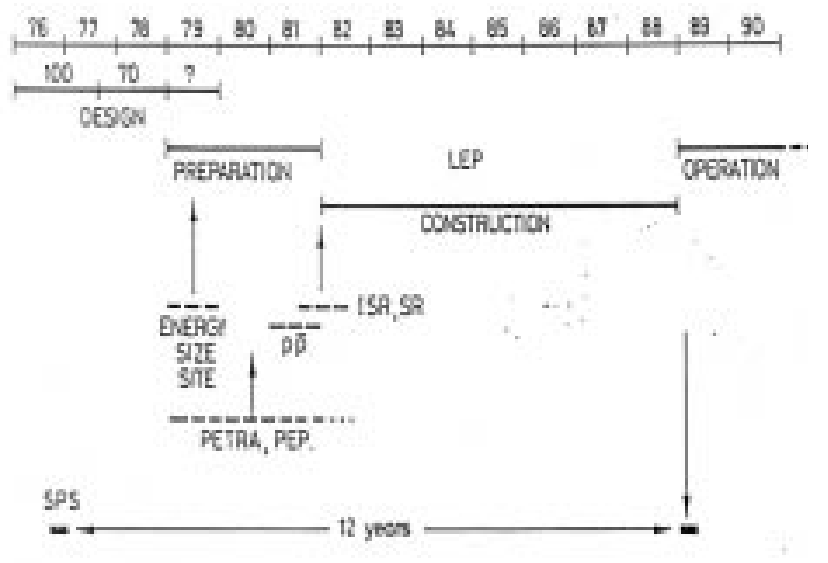

Figure 2: John Adams' first LEP construction plan it was even "the least probable", but its truth or not "will be answered by LEP and only by LEP"; (2) the scenario of bigger or smaller gauge groups than $\mathrm{SU} 3 \times \mathrm{SU} 2 \times \mathrm{U} 1$, e.g. the possibility of replacing $\mathrm{Z}$ exchange by exchange of pairs of heavy scalars (i.e. no Z!); (3) the 'many families' scenario allowing "neutrino wipeout" (i.e. no visible Z!); and (4) complete surprise, which he considered "the most likely" outcome. The report discussed Technicolor, but had only half a sentence on SUSY ("new R-hadrons"). 
By the time LEP actually started operating in 1989, as most remarkably foreseen by

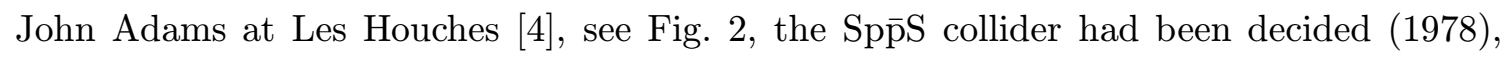
commissioned (1981), and had found the W and Z (1983); PETRA/PEP had seen clear gluon jets (1979); CLEO had seen open bottom (1981), and observation of the b lifetime had given a key stimulus to vertex detectors at LEP; but no new leptons or quarks had been found in PETRA/PEP/Tristan's factor $\sim 5$ in $\mathrm{E}_{\mathrm{cm}}$.

The scene at LEP's dawn was nicely encapsulated by Don Perkins in his ICHEP88

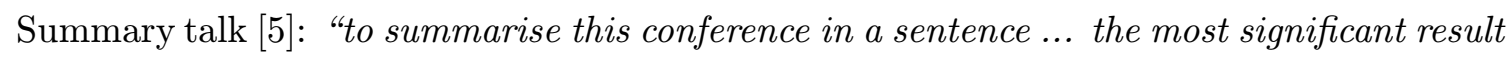
was that, against all the odds and despite our most intensive efforts to find a discrepancy somewhere, the Standard Model has somehow managed to remain intact for one more year; it is now 15 years old", which was "quite miraculous". Frank Sciulli in his LP89 Summary [i, [i.] commented "We find neither anomalies nor new particles; worse, we haven't a clue yet where they will emerge".

\section{ELECTROWEAK PHYSICS [7]}

ElectroWeak (EW) physics was the main aim of LEP from the start. In fact the SLC

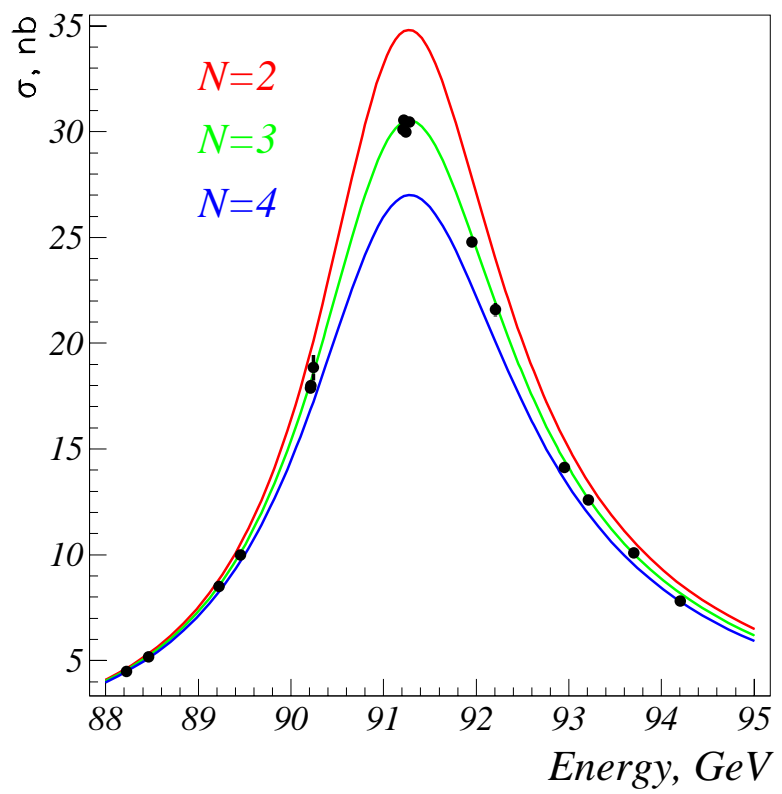

Figure 3: The final Z line-shape measurement compared with the SM predictions for 2, 3 and 4 light (compared with $\mathrm{m}_{\mathrm{Z}}$ ) neutrinos

just beat LEP to the $\mathrm{Z}$ and gave results based on 233 events at LP89 which by midNovember (after an earthquake had stopped the SLC) had been updated to 480 events giving [8i] $\mathrm{m}_{\mathrm{Z}}=91.14 \pm 0.12 \mathrm{GeV}, \Gamma_{\mathrm{Z}}=2.42_{-0.35}^{+0.45} \mathrm{GeV}$ and $\mathrm{N}_{\nu}=2.8 \pm 0.6$, which compared with $\mathrm{m}_{\mathrm{Z}}=90.9 \pm 0.35 \mathrm{GeV}$ and $\Gamma_{\mathrm{Z}}=3.8 \pm 1.5 \mathrm{GeV}$ from the CDF and UA2 results quoted at LP89 [京] 

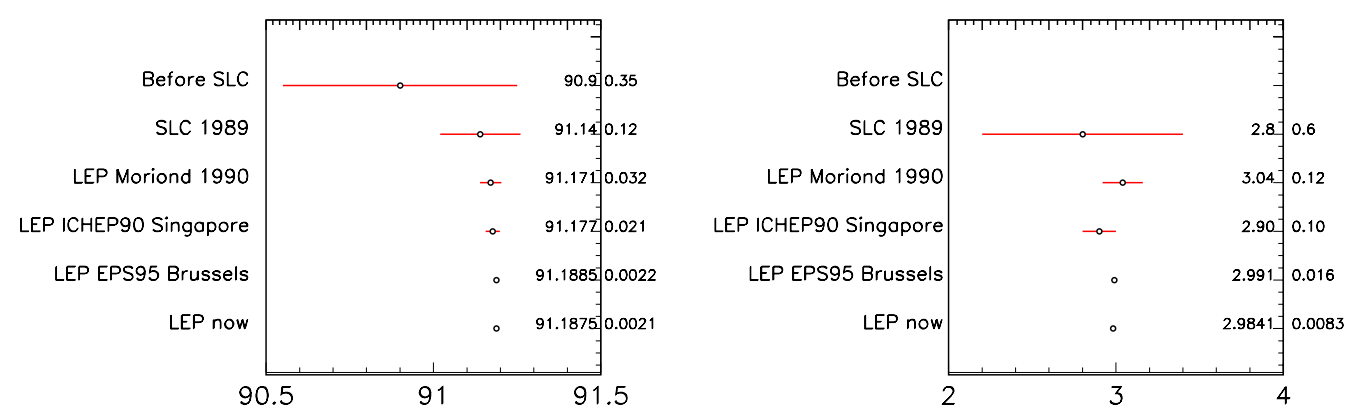

Figure 4: $\mathrm{Z}$ mass and number of neutrinos history plots

But LEP soon caught up. The first physics run ended in mid-November and the results were published within days. The results were combined at Moriond 1990 [9i] : $\mathrm{m}_{\mathrm{Z}}=91.171$ $\pm 0.012(\exp ) \pm 0.032$ (tot inc $\left.\mathrm{E}_{\mathrm{LEP}}\right) \mathrm{GeV}, \Gamma_{\mathrm{Z}}=2.538 \pm 0.026(\exp ) \pm 0.028$ (tot) $\mathrm{GeV}, \sigma_{0}$ $=40.88 \pm 0.04(\exp ) \pm 0.06$ (inc $\sigma_{\text {Bhabha }} \&$ ISR) $\mathrm{nb}$, and $\mathrm{N}_{\nu}=3.04 \pm 0.12$ (tot). The latter number was the first solid evidence ${ }^{1}$ of only 3 generations.

But, as the quoted systematic errors make clear, measuring the $\mathrm{Z}$ much better depended on progress in theory and in understanding the energy of LEP. This progress was made (see Fig. $\left.\overline{4}_{1}^{\overline{1}}\right)$ : in all, LEP improved the precisions on the $\mathrm{Z}$ mass, $\mathrm{Z}$ width, and number of neutrinos, by 2 orders of magnitude.

Currently the inputs to EW calculations are $\mathrm{m}_{\mathrm{Z}}$, now known to 24 parts per million (ppm) from the final $\mathrm{Z}$ line-shape measurement, Fig. '3i', and dependent mainly on the LEP energy, a triumph for the LEP Energy Working Group (WG); $\mathrm{G}_{\mathrm{F}}$, known from $\mu$

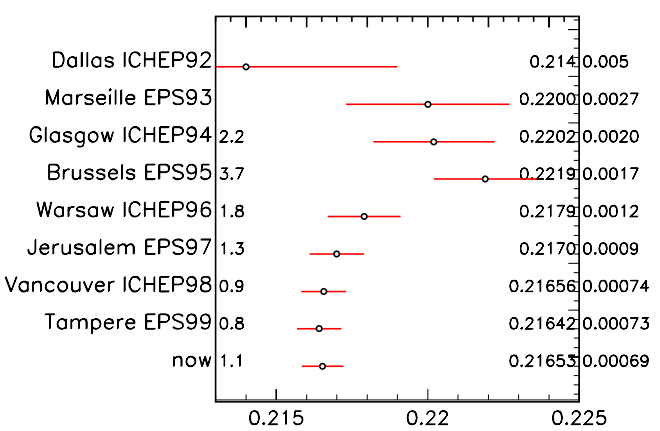
decay to $9 \mathrm{ppm} ; \alpha_{\mathrm{em}}\left(\mathrm{m}_{\mathrm{Z}}\right)$ which, because of

Figure 5: Time dependence of the $\mathrm{R}_{\mathrm{b}}$ measurement: value and error on right, number of standard deviations ( $\sigma$ 's) from the SM on left hadronic corrections, is still known to only

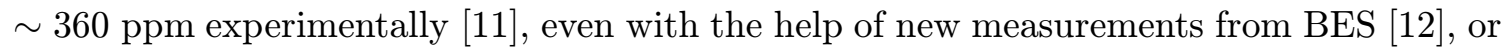
to $\sim 200 \mathrm{ppm}$ using additional perturbative QCD constraints [133]; $\mathrm{m}_{\text {top }}$, once an unknown, now known to $\pm 3 \% ; \mathrm{m}_{\text {Higgs }}$, now the only unknown (fitted) parameter; and $\alpha_{\mathrm{s}}\left(\mathrm{m}_{\mathrm{Z}}\right.$ ), now known to $\sim(2-3) \%$. Of these, $\mathrm{m}_{\mathrm{Z}}, \mathrm{G}_{\mathrm{F}}$, and $\alpha_{\mathrm{em}}\left(\mathrm{m}_{\mathrm{Z}}\right)$ enter at tree level; $\mathrm{m}_{\text {top }}$ and $\mathrm{m}_{\text {Higgs }}$ enter in EW loop corrections; $\alpha_{\mathrm{s}}\left(\mathrm{m}_{\mathrm{Z}}\right)$ enters in $\mathrm{QCD}$ corrections.

The correct prediction of $\mathrm{m}_{\text {top }}$ was a major triumph for the Standard Model. At EPS93

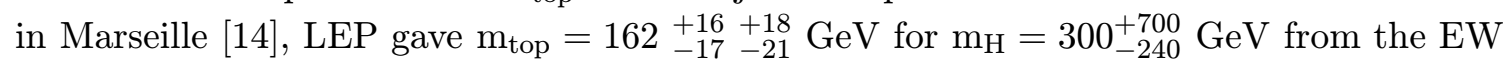
WG SM fit. At ICHEP94 in Glasgow [1] alone, $171_{-12}^{+11}{ }_{-19}^{+18} \mathrm{GeV}$ adding $\mathrm{p} \overline{\mathrm{p}}+\nu$ data, and $178_{-11}^{+11}{ }_{-19}^{+18} \mathrm{GeV}$ adding $\mathrm{A}_{\mathrm{LR}}$ from the

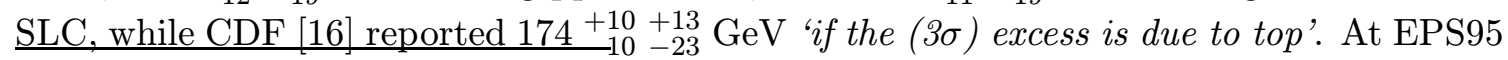

${ }^{1}$ the story of the cosmological limit available at around that time is told in Ref. [i10] 
Preliminary
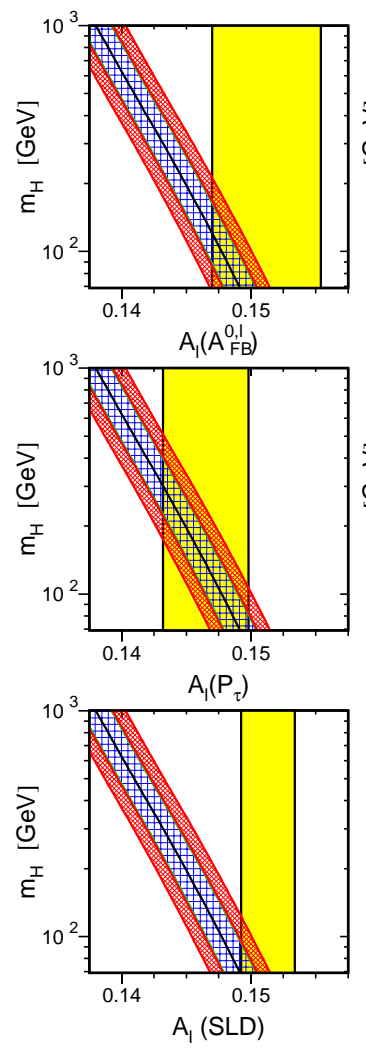

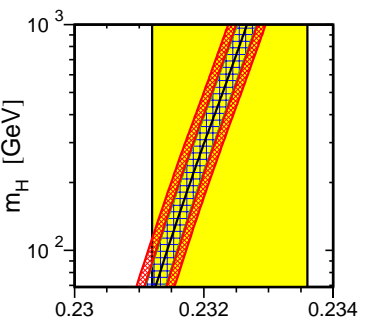

$\sin ^{2} \Theta_{\text {eff }}^{\text {lept from }<Q_{F B}>}$

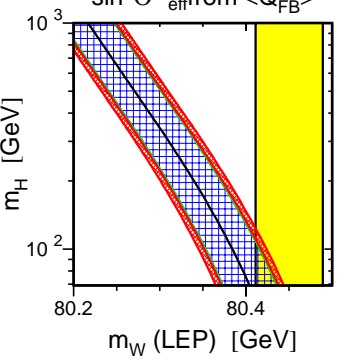

Measurement

$\Delta \alpha_{\text {had }}^{(5)}=0.02761 \pm 0.00036$

$\alpha_{\mathrm{s}}=0.118 \pm 0.002$

\#曲 $m_{\mathrm{t}}=174.3 \pm 5.1 \mathrm{GeV}$
Preliminary
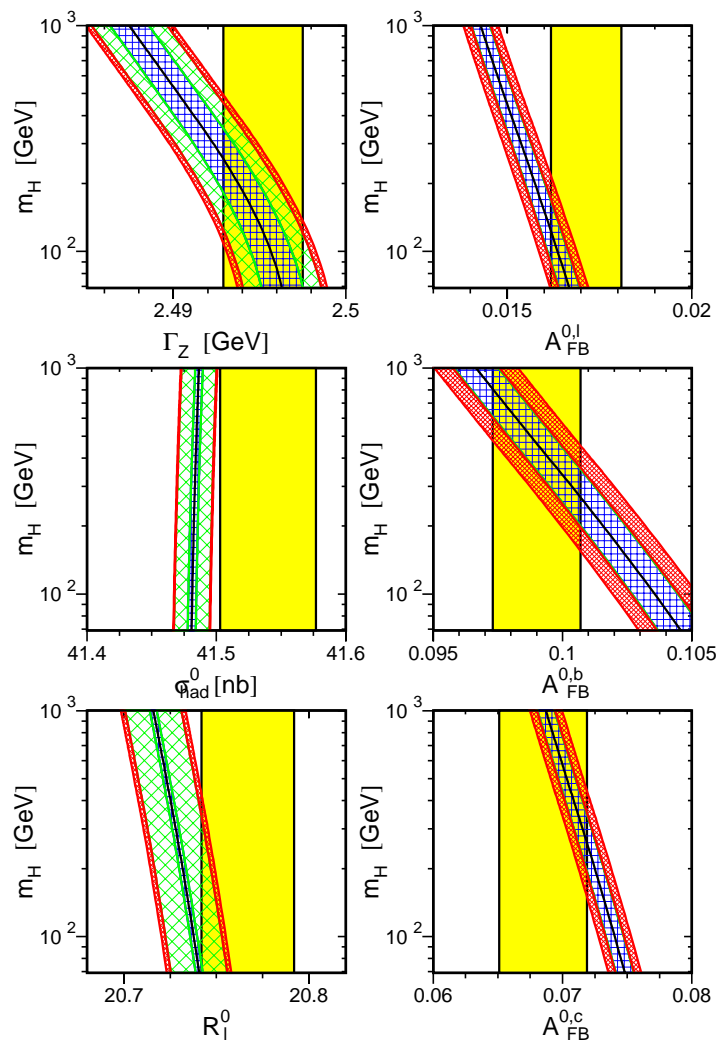

Figure 6: Higgs mass predictions, shown by the multi-coloured bands where the different colours show the different sources of uncertainty as indicated, compared with individual EW measurements, shown by the vertical $\pm 1 \sigma$ yellow bands

in Brussels, CDF and D0 both finally reported top's 'observation' [i]

Its second triumph, though of a different kind, was the ' $R_{b}-R_{c}$ crisis'. By ICHEP92 in Dallas the $\mathrm{Z}^{0} \rightarrow \mathrm{b} \overline{\mathrm{b}}$ partial width $\Gamma_{\mathrm{b}}$ had been measured as $373 \pm 9 \mathrm{MeV}$ in good agreement with the SM [i] $1 \overline{8}$. This was the solidest available evidence that top really had to exist, predictions being $\sim 370 \mathrm{MeV}$ for $\mathrm{I}_{3}^{\mathrm{b}}=-\frac{1}{2}$, and $24 \mathrm{MeV}$ for $\mathrm{I}_{3}^{\mathrm{b}}=0$ [1] $\left.{ }_{1}^{\overline{9}}\right]$. Pushing techniques to the limits improved precision rapidly until, at EPS95 in Brussels, the (in)famous ' $R_{b}, R_{c}$ crisis' appeared [201] : due to an apparently small change in the numbers when $R_{c}$ was also

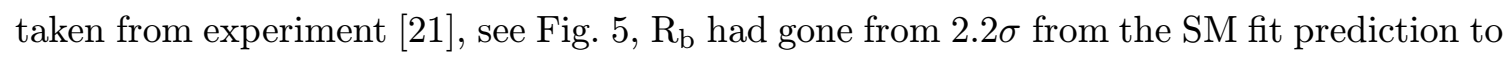
$3.7 \sigma$. In Supersymmetry (SUSY), this could have signalled light stops or charginos which would be seen soon at LEP1.5 or LEP2 [2̄22i]. So a big experimental effort was made. But the anomaly went away, and direct searches at 130-136 and 161-172 GeV found nothing. This was really very sad, but the work done on b-tagging in support of all this effort was very important later for the LEP2 Higgs search.

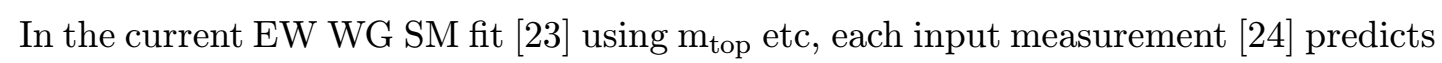

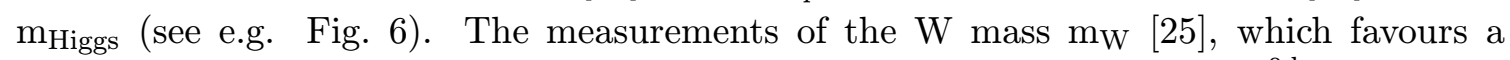
particularly low Higgs mass, and of the forward-backward b asymmetry $A_{\mathrm{FB}}^{0, b}[2 \overline{2} \overline{6}]$, which 


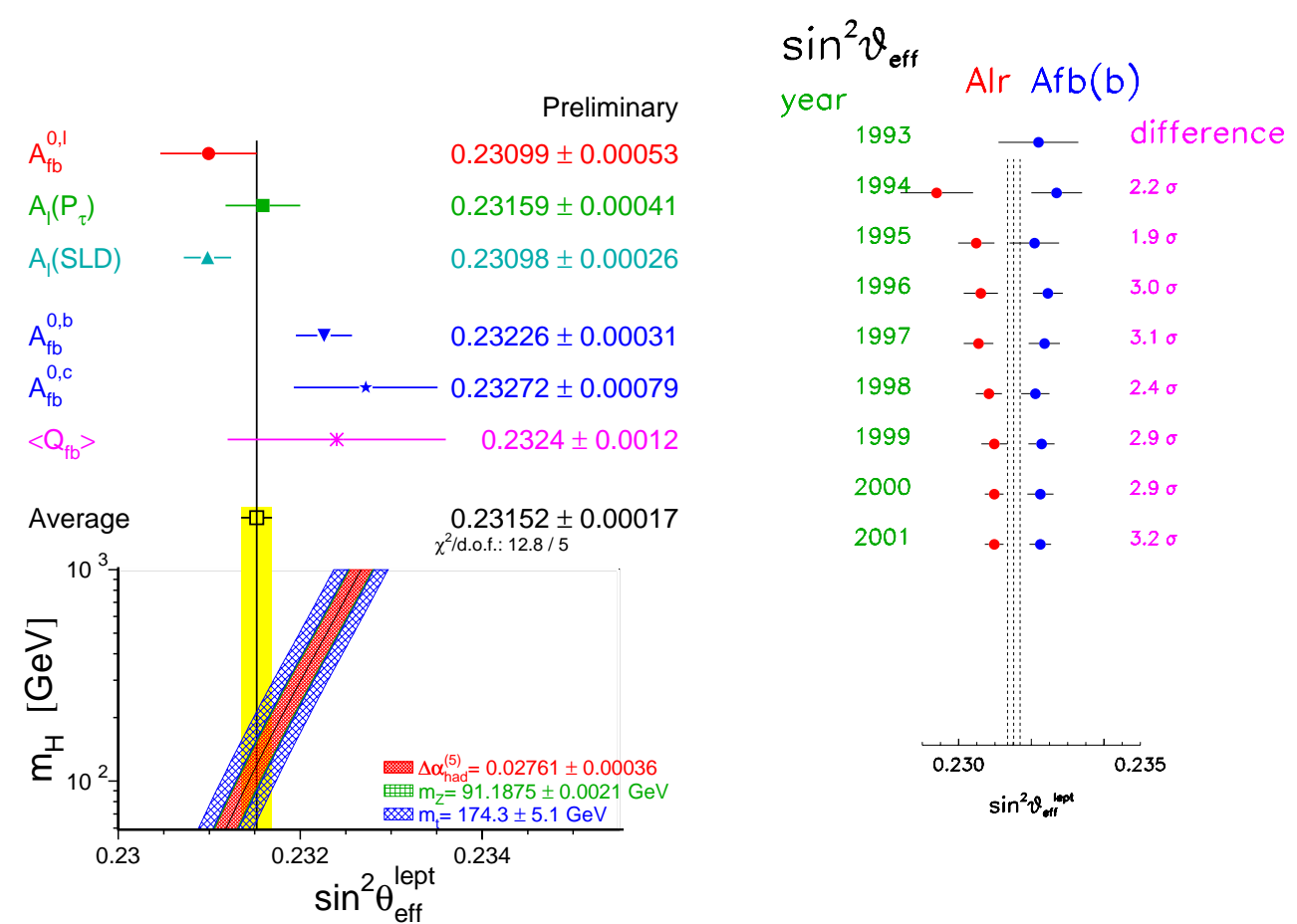

Figure 7: Comparison/combination of different measurements of $\sin ^{2} \theta_{\text {eff }}^{\text {lept }}$ (left); and comparison of the two most precise values, those from SLAC's left-right polarisation asymmetry and LEP's forward-backward b asymmetry, as a function of time (right)
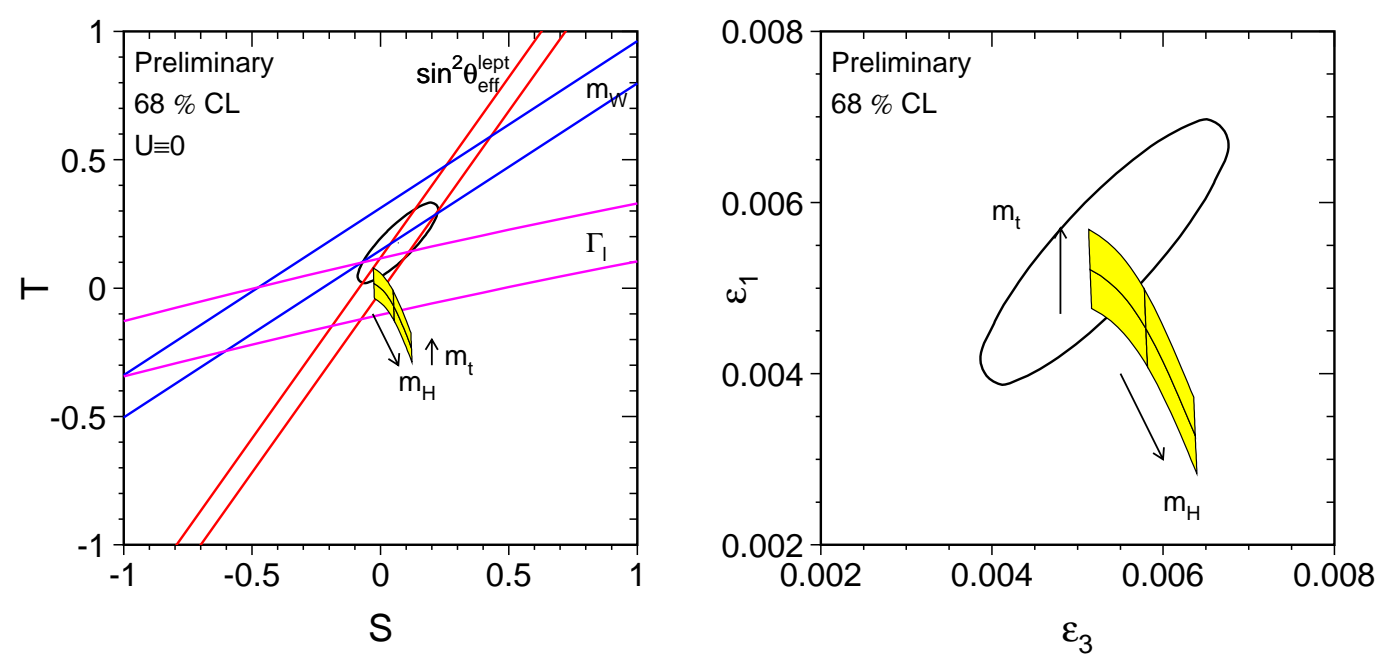

Figure 8: The S-T plot for $\mathrm{U}=0.0$ (left); and the $\sim$ equivalent $\epsilon_{1} \epsilon_{3}$ plot with $\epsilon_{2}$ also free so that it absorbs the $\mathrm{W}$ mass measurement (right)

favours a particularly high Higgs mass, are still very much ongoing.

In the SM, the various asymmetry and polarisation measurements can be considered 

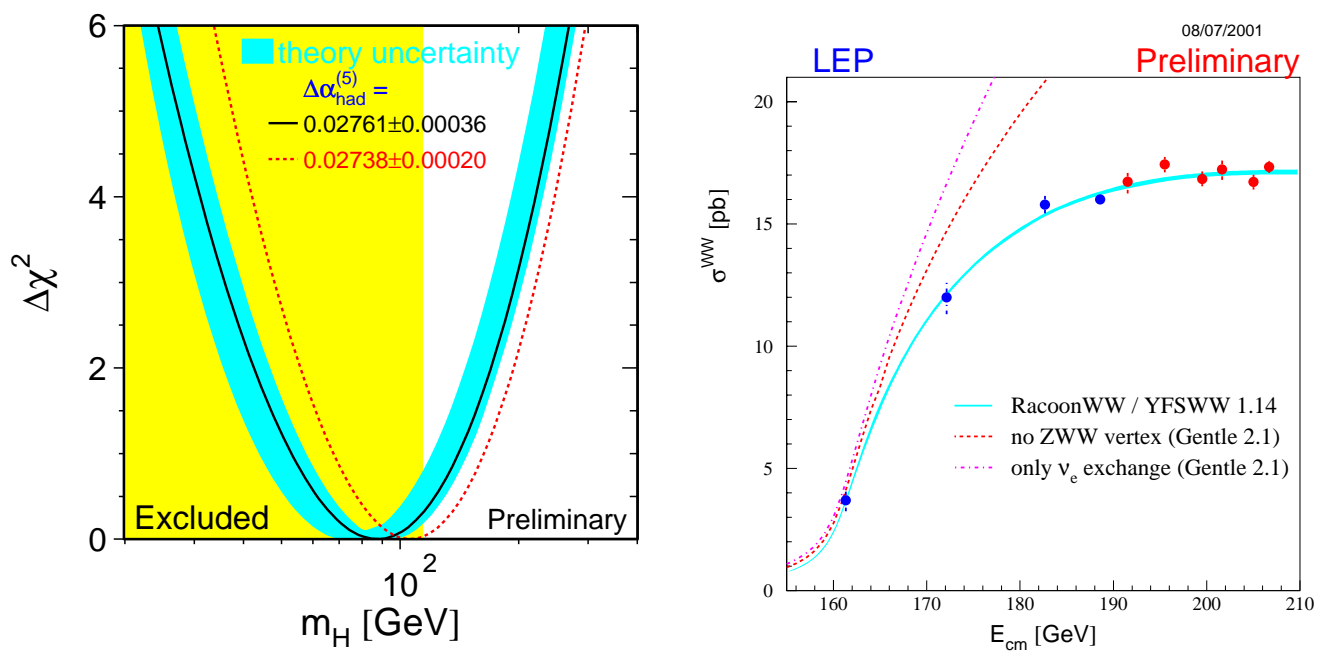

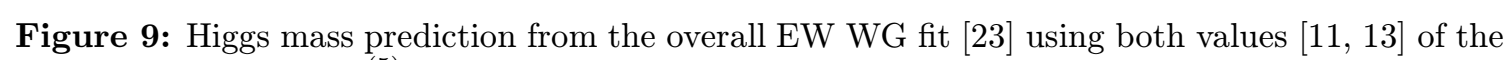
hadronic correction $\Delta \alpha_{\text {had }}^{(5)}$ to $\alpha_{\mathrm{em}}\left(\mathrm{m}_{\mathrm{Z}}\right)$ (left); and the total $\mathrm{e}^{+} \mathrm{e}^{-} \rightarrow \mathrm{W}^{+} \mathrm{W}^{-}$cross section compared with the full SM prediction, which was recently revised [32i] to match the high precision of the data, and the predictions without the ZWW vertex and with only the $\nu_{\mathrm{e}}$ exchange diagram (right)

as measurements of the same quantity, the effective weak mixing angle $\sin ^{2} \theta_{\text {eff }}^{\text {lept }}$. Their comparison and combination are shown in Fig. inist (left). The two most precise values, those from LEP's forward-backward b asymmetry [2] $2 \overline{6}$ ] and SLAC's left-right polarisation

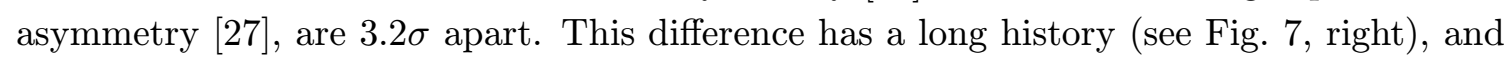
has given rise to various speculations [i2 $\overline{2} \overline{8}]$, but as 20 different quantities are fitted the purely statistical probability of finding one such disagreement is not so small. The $\operatorname{Prob}\left(\chi^{2}\right)$ of the $\sin ^{2} \theta_{\text {eff }}^{\text {lept }}$ fit is $2.5 \%$, that of the overall EW WG SM fit is $8.5 \%$.

Vacuum polarisation effects are sensitive to any new physics that implies new weaklyinteracting particles. Within rather general conditions, just three independent vacuum polarisation contributions are possible, and the so-called S, T and U variables [20.20 constructed to reveal them. In addition, the U variable is close to zero in most SM extensions. Thus the S-T plot, on which each input measurement defines a band, see Fig. $\bar{p}_{1}^{\prime}$ (left), largely summarises the very tight New Physics constraints that can be deduced from these precision measurements and is a major element of "LEP's Legacy". Most SM extensions that were popular earlier, e.g. standard Technicolour, fail the S-T test. But SUSY survives because the SUSY particles effectively 'decouple' if their masses are slightly above the direct observational limits. An alternative and largely equivalent formulation ${ }^{2}$ using the $\epsilon$ parameters [30in to the $\mathcal{O}(10 \%)$ level. Other limits on new physics are coming from comparing high-energy

\footnotetext{
${ }^{2}$ the equivalence is that $\epsilon_{1} \leftrightarrow \mathrm{T}, \epsilon_{2} \leftrightarrow \mathrm{U}, \epsilon_{3} \leftrightarrow \mathrm{S}$, apart from scale factors and choice of origin: by definition the STU parameters are zero for the SM predictions with certain specified values of all the relevant parameters, while the $\epsilon_{i}$ are defined to be zero for EW theory at tree-level plus pure QED+QCD corrections - and the $\epsilon_{i}$ therefore measure the purely weak loop corrections
} 
fermion and photon pair production with SM predictions [3inin].

The SM Higgs mass predicted by the full EW WG SM fit, see Fig. $\underline{p}_{1}$ (left), is $\mathrm{m}_{\text {Higgs }}=88_{-35}^{+53}$ $\mathrm{GeV}$, or $\mathrm{m}_{\mathrm{Higgs}}<196 \mathrm{GeV}$ at $95 \% \mathrm{cl}$. Another classic plot, clearly and precisely confirming the large cancellation between WW production amplitudes required by gauge theory, one

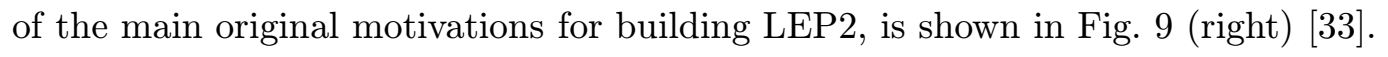

The ZZ and other 4-fermion production cross-sections and the possible anomalous gauge-invariant couplings of the $\mathrm{W}$ and $\mathrm{Z}$ are also being studied [i] $\left.\overline{3}^{4}\right]$.

\section{HEAVY FLAVOUR PHYSICS}

This was NOT in the original 1970's LEP program (b and $\tau$ were $\sim$ unknown then!). But LEP began a new era based on Silicon Vertex Detectors.

The mean $b$ lifetime has been measured with great enthusiasm ever since the start of LEP. The $\mathrm{B}_{\mathrm{s}}^{0}, \Lambda_{\mathrm{b}}^{0}$ and $\Xi_{\mathrm{b}}$ were identified first at LEP. The individual $\mathrm{B}_{\mathrm{u}}^{+}, \mathrm{B}_{\mathrm{d}}^{0}, \mathrm{~B}_{\mathrm{s}}^{0}$ and

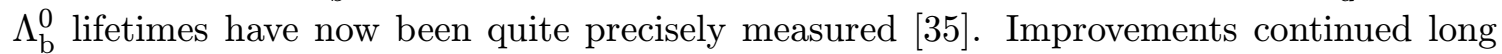
after LEP1 data-taking stopped in 1995. Babar and Belle have now entered the game for $\mathrm{B}_{\mathrm{d}}^{0}$ and $\mathrm{B}^{-}$, but the most precise $\mathrm{B}_{\mathrm{d}}^{0}$ and $\mathrm{B}^{-}$lifetimes are still the brand-new ones from LEP. The observed b-meson lifetime hierarchy shows that the spectator model is indeed violated at the $\sim 10 \%$ level as expected, but there is arguably a problem with the b-baryon lifetime. Whether or not this is a job for theory should be answered soon by CDF/D0.
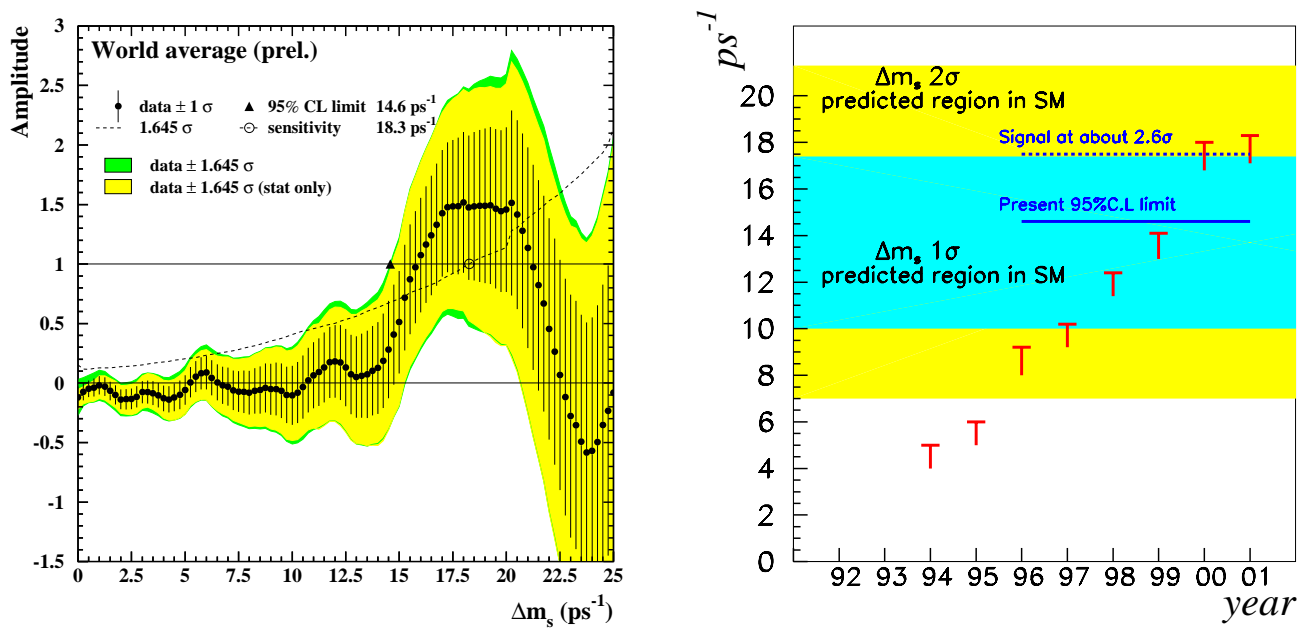

Figure 10: $\mathrm{B}_{\mathrm{s}}^{0}$ oscillation amplitude as a function of $\Delta \mathrm{m}_{\mathrm{s}}$ (left); and time-evolution of the $\Delta \mathrm{m}_{\mathrm{s}}$ reach (i.e. of the expected limit) compared with the $\Delta \mathrm{m}_{\mathrm{s}}$ value expected at $68 \%$ and $95 \%$ c.l. by combining other measurements of the sides of the unitarity triangle

Lifetime detection is also the key to all other LEP B physics. Many studies involved enormous effort, like the study of semi-leptonic decays [i] $\left.{ }^{3} \overline{6}\right]$ w where LEP had to work very

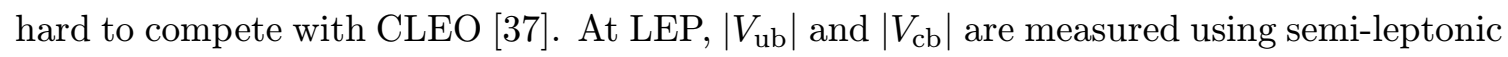
decays: $\left|V_{\mathrm{ub}}\right|$ from inclusive spectra in $b \rightarrow u$ enriched decays, and $\left|V_{\mathrm{cb}}\right|$ from inclusive semileptonic \& $\overline{\mathrm{B}_{\mathrm{d}}^{0}} \rightarrow \mathrm{D}^{\star+} \ell^{-} \overline{\nu_{\ell}}$ decays. $\left|V_{\mathrm{ub}}\right| /\left|V_{\mathrm{cb}}\right|$ determines the length of the left-hand-side 


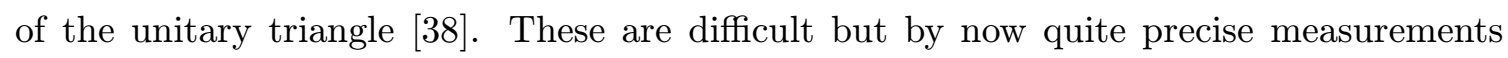
which also have very challenging theory uncertainties that are being reduced with help from theorists.

$\mathrm{B}_{\mathrm{d}, \mathrm{s}}^{0}-\overline{\mathrm{B}_{\mathrm{d}, \mathrm{s}}^{0}}$ oscillations are a central theme of LEP B physics [i] $\left.\overline{3}_{\overline{9}}\right]$. The time dependences were first seen at LEP. The oscillation frequency, $\Delta m_{q}$, is proportional to $\left|V_{t q}\right|^{2}$ :

$$
\Delta m_{q}=\frac{G_{F}^{2} m_{W}^{2}}{6 \pi^{2}} \eta_{B} S\left(m_{t}^{2} / m_{W}^{2}\right) m_{B_{q}} \hat{B}_{B_{q}} f_{B_{q}}^{2}\left|V_{t q}\right|^{2}
$$

and the substantial QCD uncertainty in $\hat{B}_{B_{q}} f_{B_{q}}^{2}$ largely cancels in the ratio $\Delta m_{d} / \Delta m_{s}$,

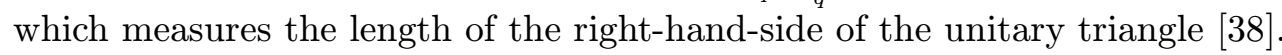
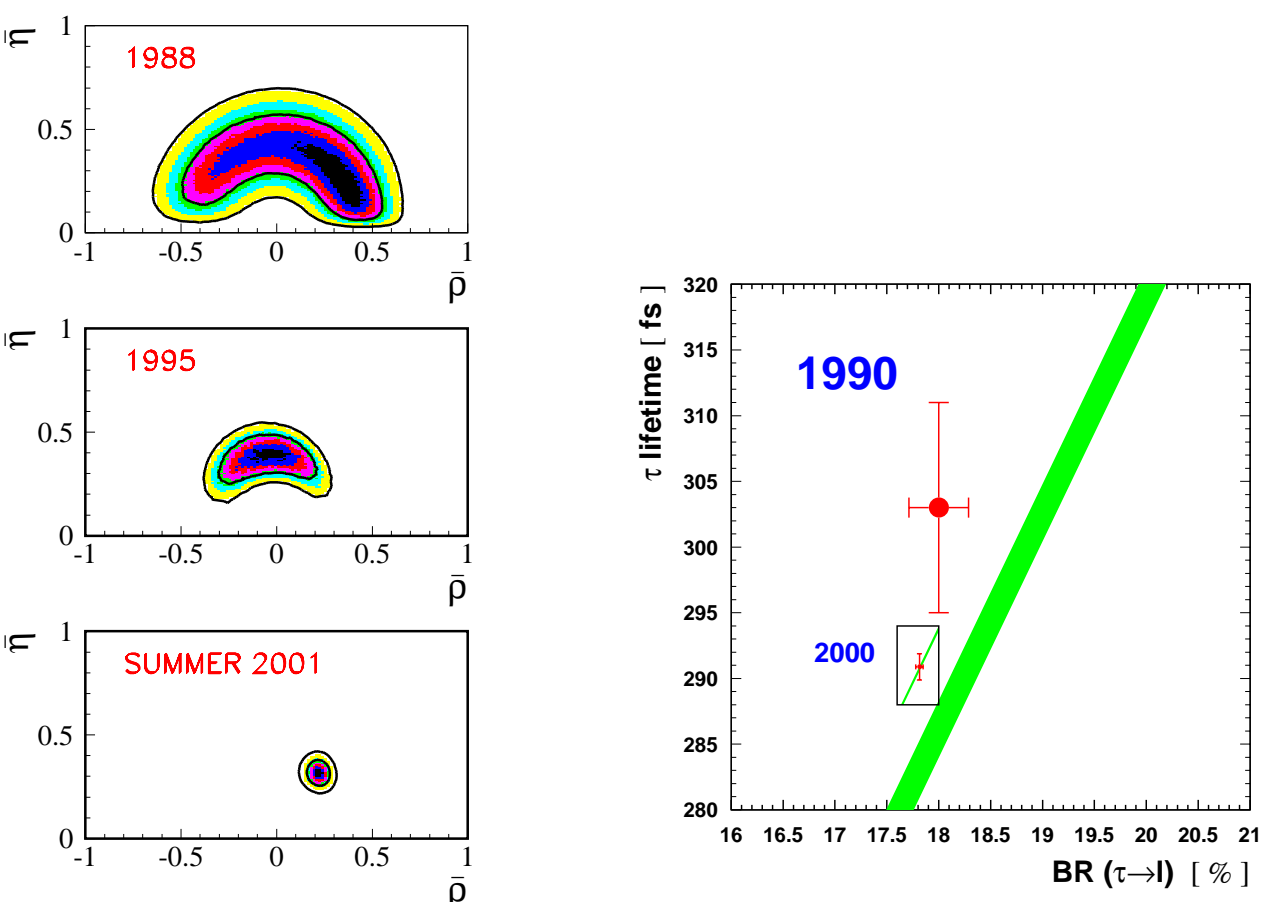

Figure 11: Time evolution of the position of the vertex of the unitarity triangle in the $\bar{\rho}, \bar{\eta}$ plane since 1988 (left); and the improvement in the test of e, $\mu, \tau$ universality since 1990 (right)

$\Delta m_{d}$ is measured at LEP to $3 \%$ precision, $0.484 \pm 0.015 \mathrm{ps}^{-1}$, and again the most precise value is a brand-new one, but Babar and Belle now dominate the world average of $0.489 \pm 0.008 \mathrm{ps}^{-1}$. Currently, see Fig. '10 $\overline{0}_{1}^{\prime}(\mathrm{left}), \mathrm{B}_{\mathrm{s}}$ oscillation results combined using the amplitude method give only $\Delta m_{s}>14.6 \mathrm{ps}^{-1}$ at $95 \% \mathrm{cl}$, despite an expected limit of 18.3 $\mathrm{ps}^{-1}$, because of a $2.6 \sigma$ signal-like effect at $17 \mathrm{ps}^{-1}$, near the value expected. At such high $\Delta m_{s}$, LEP and SLD have equal weight. Continuing improvements in analysis techniques, particularly in improving sample purity and proper time resolution, have pushed the $\Delta m_{s}$ reach far beyond what was thought possible and well into (indeed almost beyond!) the most interesting range, see Fig. '10 $10_{1}^{i}$ (right). So one should keep watching this plot! (but, sadly, perhaps now mainly for the effect of CDF/D0's Run II data). 


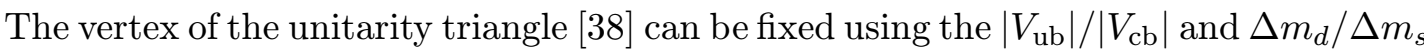
(and $\epsilon_{K}$ ) world averages, see Fig. i1 $\overline{1}_{1}^{\prime}$ (left). The result obtained, $\sin 2 \beta=0.696 \pm 0.067$, or $0.678_{-0.101}^{+0.078}$ without $\epsilon_{K}$, at least gives BaBar, Belle and CDF/D0 something to aim at!

Turning to $\tau$-decays [i $\left[\begin{array}{l}4 \\ 0\end{array}\right]$, any one of the LEP expts would dominate the present world average $\tau$ lifetime. Their much improved $\tau$ branching ratios consigned the infamous ' 1 prong' anomaly ( $>4 \sigma$ clash between exclusive and inclusive 1-prong BR's) to a footnote in history. Combining their lifetimes and branching ratios tested $e, \mu, \tau$ universality in $\tau$ decays an order-of-magnitude more precisely than before, but universality survived because

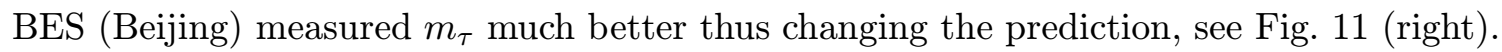

\section{QCD}

QCD is beautiful parameterless (in principle) theory giving strong coupling at the hadron scale and becoming asymptotically free at high energy. Before LEP startup its experimental support was already solid, quantitative and impressive. Measurements in a wide range of processes: $\mathrm{R}_{\mathrm{e}^{+} \mathrm{e}^{-}}, \mathrm{e}^{+} \mathrm{e}^{-}$event shapes, $\Upsilon$ decays, deep inelastic scattering, and photon structure functions had given an overall result of $\alpha_{\mathrm{S}}\left(\mathrm{m}_{\mathrm{Z}}\right) \simeq 0.11 \pm 0.01$, but no convincing

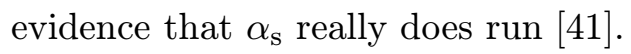
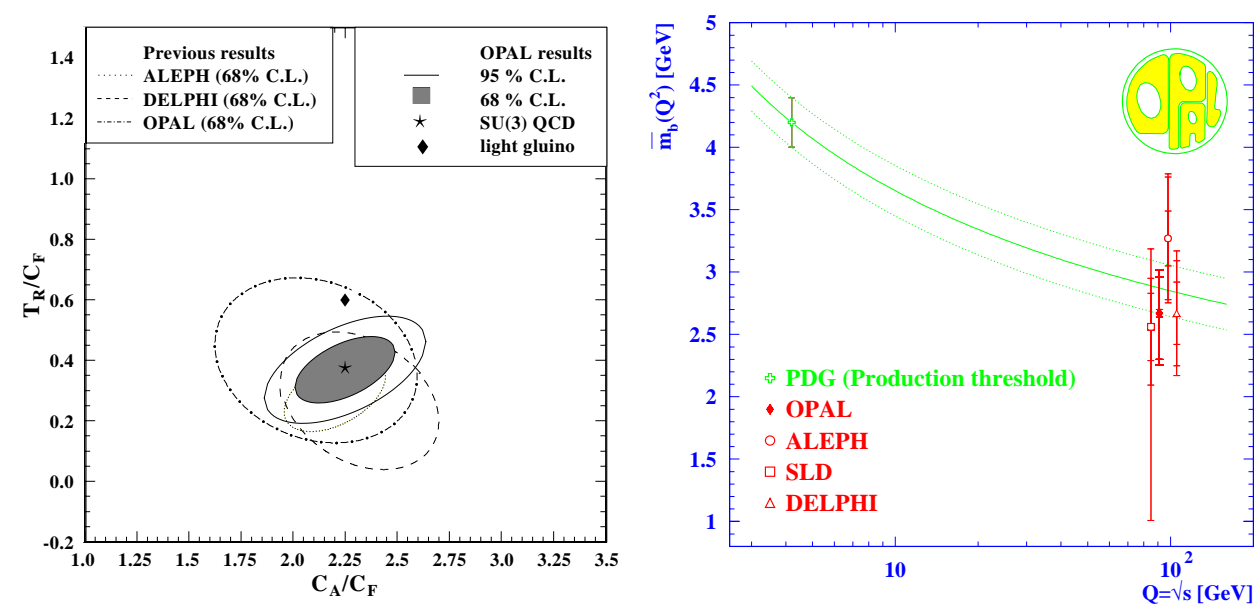

Figure 12: LEP measurements of $\mathrm{C}_{\mathrm{A}} / \mathrm{C}_{\mathrm{F}}$ and $\mathrm{T}_{\mathrm{R}} / \mathrm{C}_{\mathrm{F}}=0.5 / \mathrm{C}_{\mathrm{F}}$, where $\mathrm{C}_{\mathrm{A}}$ is the triple-gluon $g g g$ coupling and $\mathrm{C}_{\mathrm{F}}$ the quark-quark-gluon qqg coupling (left), and of the b-quark mass $\mathrm{m}_{\mathrm{b}}$ at the scale $\mathrm{m}_{\mathrm{Z}}$ compared with the value at the scale $\mathrm{m}_{\mathrm{b}}$ (right)

At LEP the higher energy, clean initial conditions and high statistics have given much greater precision and many beautiful tests and insights into the theory. Examples include (1) the precise measurement of the triple-gluon coupling from the angular distributions of

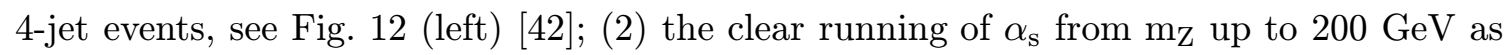
required by 'asymptotic freedom' and also down to $\sim 40 \mathrm{GeV}$ towards 'confinement' [4 $4 \overline{3}]$; and (3) the clear running of $m_{b}$ from the $m_{b}$ scale to the $m_{Z}$ scale, see Fig. '1

The value of $\alpha_{\mathrm{s}}\left(\mathrm{m}_{\mathrm{Z}}\right)$ has been measured in many ways [4 $\left.4 \overline{3} \overline{3}\right]$, but when using event shape variables the 'scale' uncertainty limits the precision. The 'scale' $\mu$ is not a real parameter 

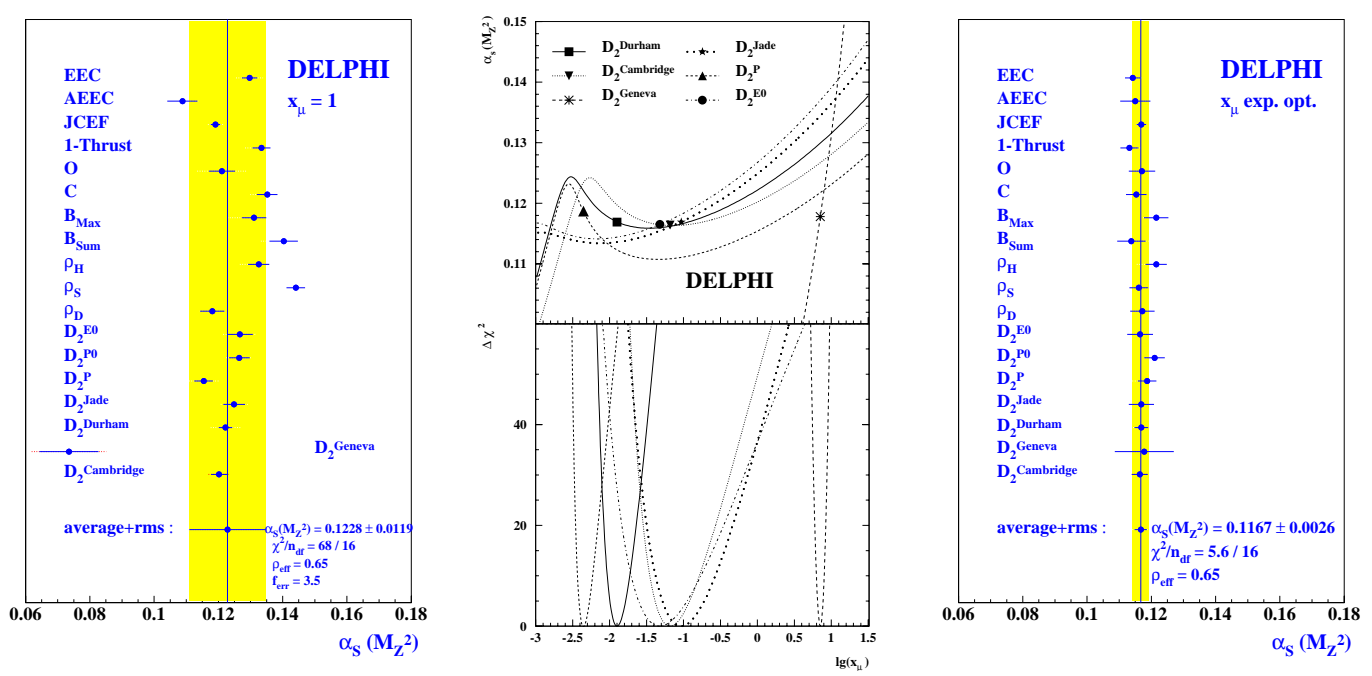

Figure 13: Values of $\alpha_{\mathrm{s}}$ obtained from event shape variables using the fixed 'theoretical' scale $\mathrm{x}_{\mu}=\mu / \sqrt{\mathrm{s}}=1$ (left) and using experimentally fitted scale values (right): the central plots show the variation of $\chi^{2}$ (lower) and $\alpha_{\mathrm{s}}$ (upper) with $\mathrm{x}_{\mu}$ in the fit. The result for the differential 2-jet rate using the 'Geneva' jet algorithm, $\mathrm{D}_{2}^{\text {Geneva }}$, is particularly impressive because $\alpha_{\mathrm{s}}$ varies so rapidly with $\mathrm{x}_{\mu}$ near the best-fit point. Plots are from [4 $\left.{ }^{4}{ }^{\prime}\right]$ except that no additional scale error is shown.

of QCD, it enters only because the perturbative expansion is cut off, so terms that contain it somehow represent the effect of the missing higher order terms. The problem is that extracting $\alpha_{\mathrm{s}}$ from different event shape variables using the theoretically-preferred fixed "physical" scale $\mathrm{x}_{\mu}=\mu / \sqrt{\mathrm{s}}=1$ gives very poor consistency (Fig. $1 \overline{3} \overline{3}_{1}^{\prime}$ left). But fitting the

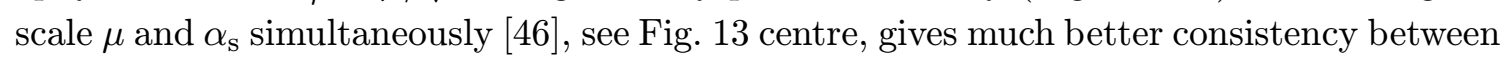
different jet-rate measurements and also between other event-shape variables - in fact the consistency is excellent between all event variables tested (Fig. II $\overline{3}_{1}^{\prime}$ right). It seems nature is trying to tell us that the $\mu$-dependences describe higher-order effects much better than expected, but this issue is not yet understood theoretically.

12 years of detailed and still ongoing study have led to excellent decriptions of jet structures, fragmentation and hadronisation effects [i] $\overline{7}]$, and the resulting superb quality of QCD event generators at LEP has been crucial in Electro-Weak studies as well as in new particle searches. Bose-Einstein and colour reconnection effects are still hot topics [4] $4 \overline{4} 8 \overline{-}$, especially in the $\mathrm{W}$ mass context, as they are not understood well yet. Good progress is now being made in the study of $\gamma \gamma$ interactions [י] ${ }_{-}^{\overline{4}} \overline{1}$.

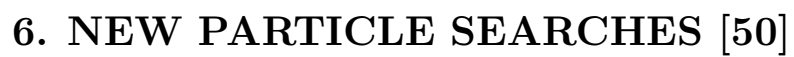

Searches at LEP benefit from a clean well-understood initial situation, high performance hermetic detectors, and (especially for the Higgs search) high-purity high-efficiency b-tags.

Before LEP, there was no SM Higgs exclusion at all for a Higgs mass $\mathrm{m}_{\mathrm{H}} \simeq 0$ or $>3.6 \mathrm{GeV}$, and in between only small bands were excluded by the absence of $\pi \rightarrow \mathrm{He} \nu$, $\mathrm{K} \rightarrow \pi \mathrm{H}$, and $\mathrm{B} \rightarrow \mathrm{XH}$ decays, etc. 
At LEP1, an SM Higgs in the mass range $0-25 \mathrm{GeV}$ was excluded already by PDG90 and Moriond90 [i] (recoiling against $\mathrm{Z}^{*}$ to $\mu^{+} \mu^{-}$), with a Higgs decaying to $e^{+} e^{-}$up to $212 \mathrm{MeV}$, or with a Higgs decaying to $\mu^{+} \mu^{-}, \tau^{+} \tau^{-}$or $q \bar{q}$ up to $25 \mathrm{GeV}$. The larger range $0-44 \mathrm{GeV}$ was excluded by ICHEP90 in Singapore [52], and 0-66 GeV by the full LEP1 data.

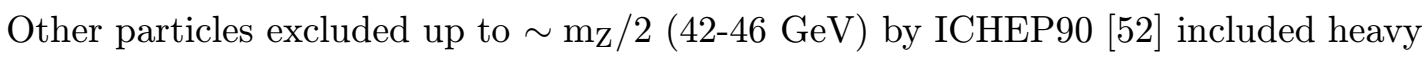
stable particles, charginos, sleptons, squarks, charged Higgses, excited and 4th family charged \& neutral leptons, a 4th family d quark, top (but the CDF limit was already at $89 \mathrm{GeV}$ ), etc., while $\Gamma_{\text {inv }}$ excluded neutralinos up to $32 \mathrm{GeV}$ unless they are decoupled from the $\mathrm{Z}$, and this limit also reached $\simeq \mathrm{m}_{\mathrm{Z}} / 2$ with the full LEP1 data.

At the Aachen workshop [54] in 1986, exploring $\mathrm{m}_{\mathrm{H}} \simeq \mathrm{m}_{\mathrm{W}}$ was considered experimentally difficult and exploring $\mathrm{m}_{\mathrm{H}} \simeq \mathrm{m}_{\mathrm{Z}}$ was even considered impossible! But it was realised later that good b-tags could break through both barriers to reach $\mathrm{m}_{\mathrm{H}} \sim \sqrt{\mathrm{s}}-100 \mathrm{GeV}$. The good b-tags came via the big efforts to measure $R_{b}$. Highly sophisticated analysis techniques combining many indicators via neural network and likelihood ratio techniques ${ }^{3}$ finally improved the reach to $\mathrm{m}_{\mathrm{H}} \sim \sqrt{\mathrm{s}}-\mathrm{m}_{\mathrm{Z}}$.

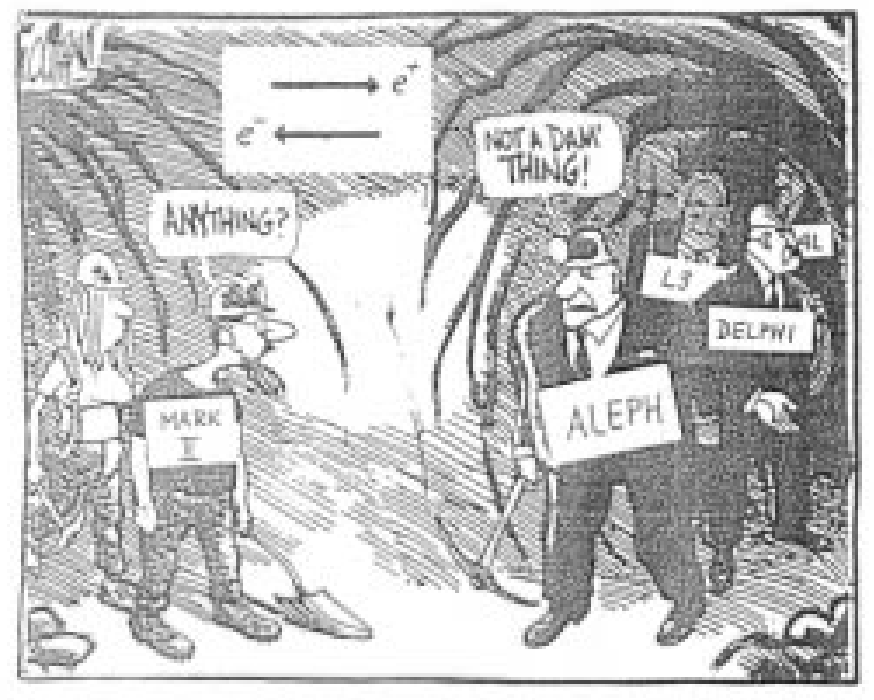

Figure 14: The final summary of LEP search results at ICHEP90 [52 2 , "taken from a Singapore newspaper and slightly adapted" and unfortunately(?) still quite appropriate

A sophisticated (and conservative!) statistical technique (the 'Modified ${ }^{4}$ Frequentist'

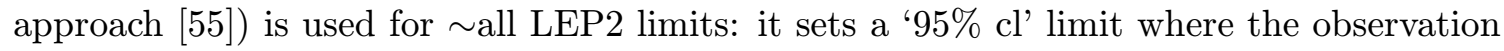
would be 20 times less frequent for the $S+B$ (i.e. signal+background) hypothesis than for background alone, not just where the frequency for the $S+B$ hypothesis falls to $5 \%$ as in the standard frequentist approach.

\footnotetext{
${ }^{3}$ in fact even with no b-tagging these techniques now break easily through both barriers and provide 'flavour-independent' limits [53] within a few $\mathrm{GeV}$ of the limits from the standard analyses

${ }^{4}$ the 'Modification', which is Bayesian-inspired, aims to prevent 'excluding' an undetectable signal, which can otherwise happen when the background fluctuates downward; the results are similar to those from standard Bayesian procedures, so the latter are sometimes used instead to avoid excessive computing
} 

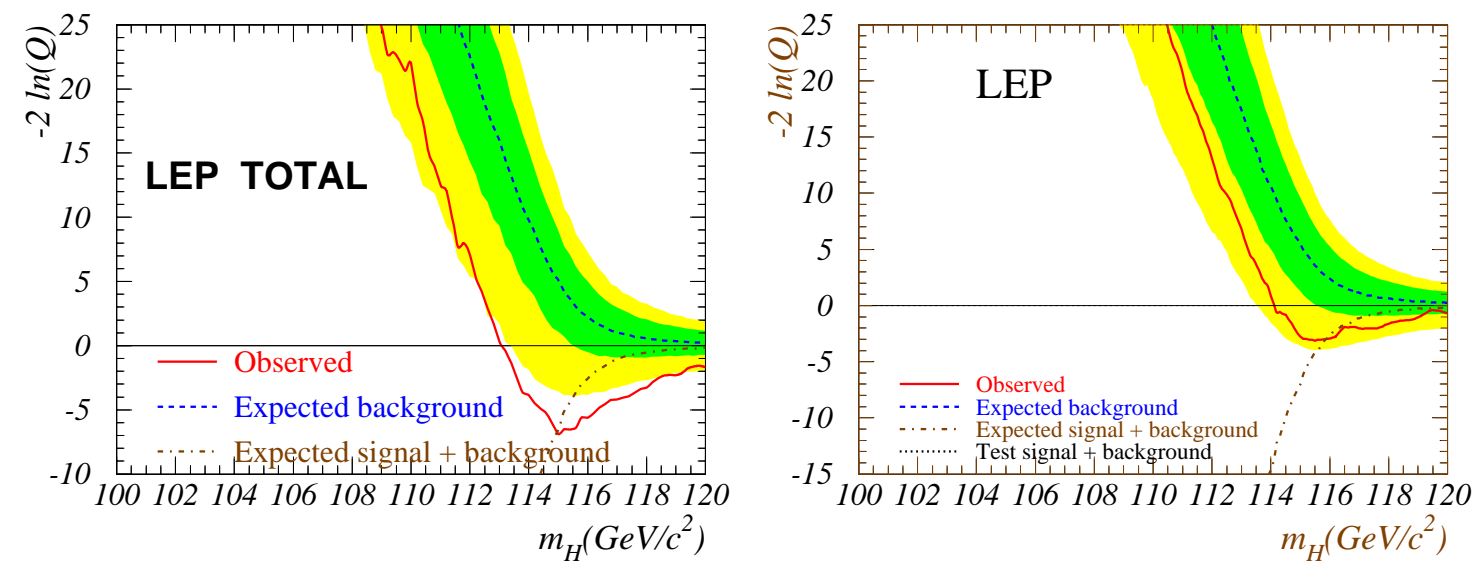

Figure 15: The value of $-2 \ln (Q)$ as a function of the hypothesised Higgs mass, where the statistical estimator $Q$ is the likelihood ratio $Q=\mathcal{L}(S+B) / \mathcal{L}(B)$, as presented at the last LEPC meeting on Nov 32000 (left) and now (right)

In the previous all-LEP Higgs search combination as presented at the last LEPC meeting (Nov 3 2000), the statistical estimator $Q=\mathcal{L}(S+B) / \mathcal{L}(B)$ agreed with the SM Higgs $\mathrm{X}$-section expected for $\mathrm{m}_{\mathrm{H}} \simeq 115 \mathrm{GeV}$, see Fig. '1 $1 \overline{5}_{1}^{1}$ (left). The probability of having observed $-2 \ln (Q) \leq-7$ at that point from a background fluctuation was evaluated to be $4.2 \times 10^{-3}$, corresponding to a $2.9 \sigma$ signal-like effect. Unfortunately, the $2.9 \sigma$ has now

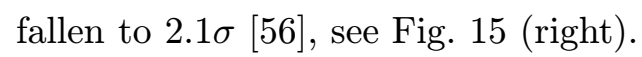

Searches for 'Exotic' new physics [5] $\overline{7}$ i] have continued vigourously at LEP2. But the other main search area is the search for SUSY. The failure of Grand Unification in the

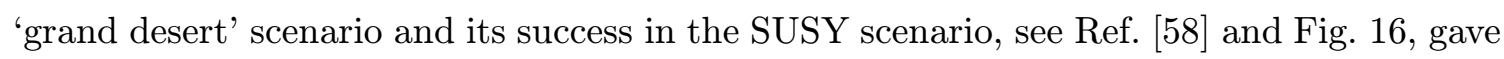
a strong positive hint. The failure was due to precision improvements due to LEP of a factor 5 in $\sin ^{2} \theta_{\mathrm{W}}$ and $\sim 3$, taken as $\sim 2$, in $\alpha_{\mathrm{s}}$; these had turned a $2 \sigma$ problem in 1987 into an $8 \sigma$ problem in 1991. Furthermore the unification mass achieved with SUSY (see Fig. 'í $\overline{1}_{1}^{\prime}$ right) was high enough to avoid problems with proton decay, and low enough to avoid problems with gravity corrections. While this didn't prove SUSY correct, because a random $2 \%$ of other SM extensions also gave acceptable unification [표효, none of them unify better than SUSY and none is independently well motivated.

But SUSY is a badly broken symmetry, and SUSY-breaking brings $\sim 124$ new constants, so a model is needed to reduce these to a handful. The SUSY-breaking model determines the production rates, mass spectrum, and decay channels of the sparticles. But there are many models - not only the popular Gravity mediated (SUGRA), Gauge Mediated (GMSB), and Anomaly Mediated (AMSB) SUSY-Breaking models, but also e.g. Gaugino Assisted Anomaly mediated, Anti-Generation-Field mediated, Brane mediated, Kaluza-Klein mediated, Radion mediated, Quantum-induced, Instanton-induced, etc. SUSY-Breaking models are on the market — each with a (slightly?) different phenomenology to look for and (slightly?) different key parameters to constrain, and each either with or without R-parity violation. 

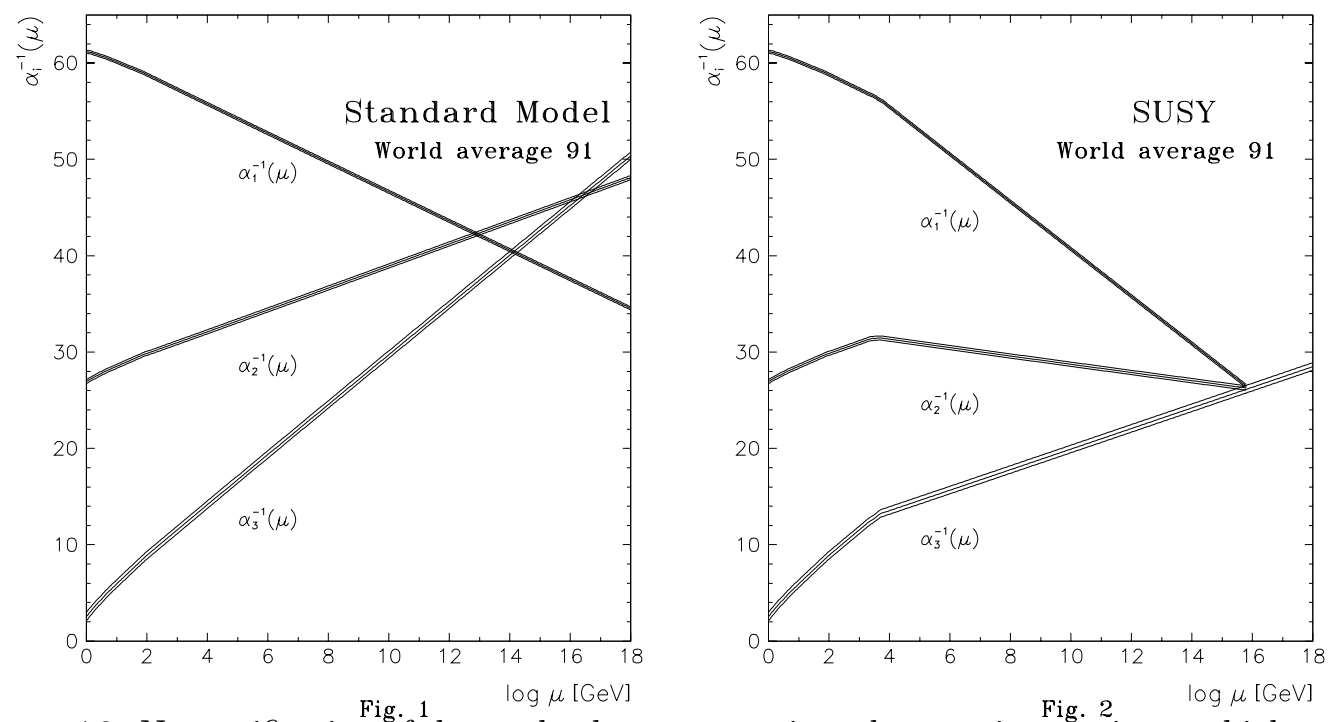

Figure 16: Non-unification of the weak, electromagnetic and strong interactions at high energy in the 'grand desert' scenario with no new physics below the unification scale (left) and their unification in the SUSY scenario (right), taken from Ref. [5

Views on SUSY cover a wide spectrum, for example:

John Schwarz [i5 $\overline{9}]$ : 'Only one message in these lectures is important for experimental research: low-energy SUSY is very well motivated theoretically, and it warrants the intense effort that is being made to devise ways of observing it'.

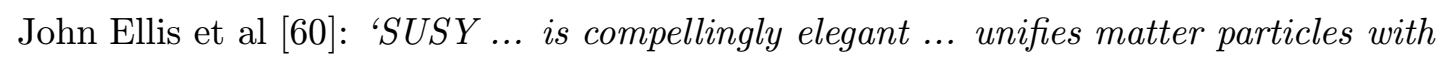
force particles ... is the only framework thought capable of connecting gravity with other interactions ... appears essential for the consistency of string theory ... and the $\chi^{0}$ is an ideal candidate for cold dark matter'.

Burt Richter [61]': 'A pure social construct ... look for supersymmetry as long as it doesn't seriously interfere with real work (top-quark, Higgs, neutrinos, etc)'.

But even to a sceptical experimenter, SUSY is at least a very useful model and motivation for numerous searches for unexpected events of various bizarre kinds that we might not have looked for otherwise (or might have been unable to publish otherwise even if we did look!).

Anyway, SUSY searches have been a major industry at LEP, even though they are very model-dependent. Most LEP searches consider near-to-minimal ('nm') versions of

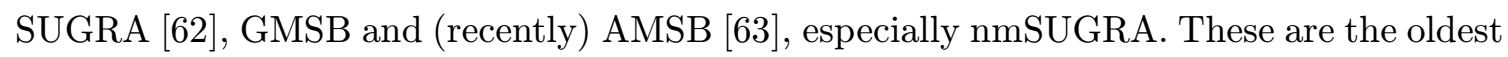
and most commonly discussed models. R-parity-conservation is usually assumed, so the Lightest Supersymmetric Particle (LSP) is stable, neutral and undetectable, and missing energy and $\mathrm{p}_{\mathrm{t}}$ are therefore key signatures, but R-parity violation (RPV) in nmSUGRA is

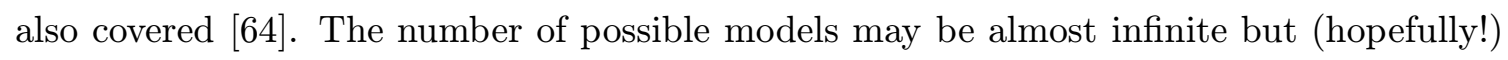
the number of relevant channels is finite and most have been explored.

Some channels enter many searches. For example the reasonably good agreements with expectations of the isolated 1-photon and 2-photon spectra, see Fig. 1'] $\mathrm{e}^{+} \mathrm{e}^{-} \rightarrow \gamma \mathrm{X}$ or $\gamma \gamma \mathrm{X}$ processes with $\mathrm{X}$ invisible, such as $\mathrm{e}^{+} \mathrm{e}^{-} \rightarrow \chi_{2} \chi_{1}$ with $\chi_{2} \rightarrow \chi_{1} \gamma$ and 

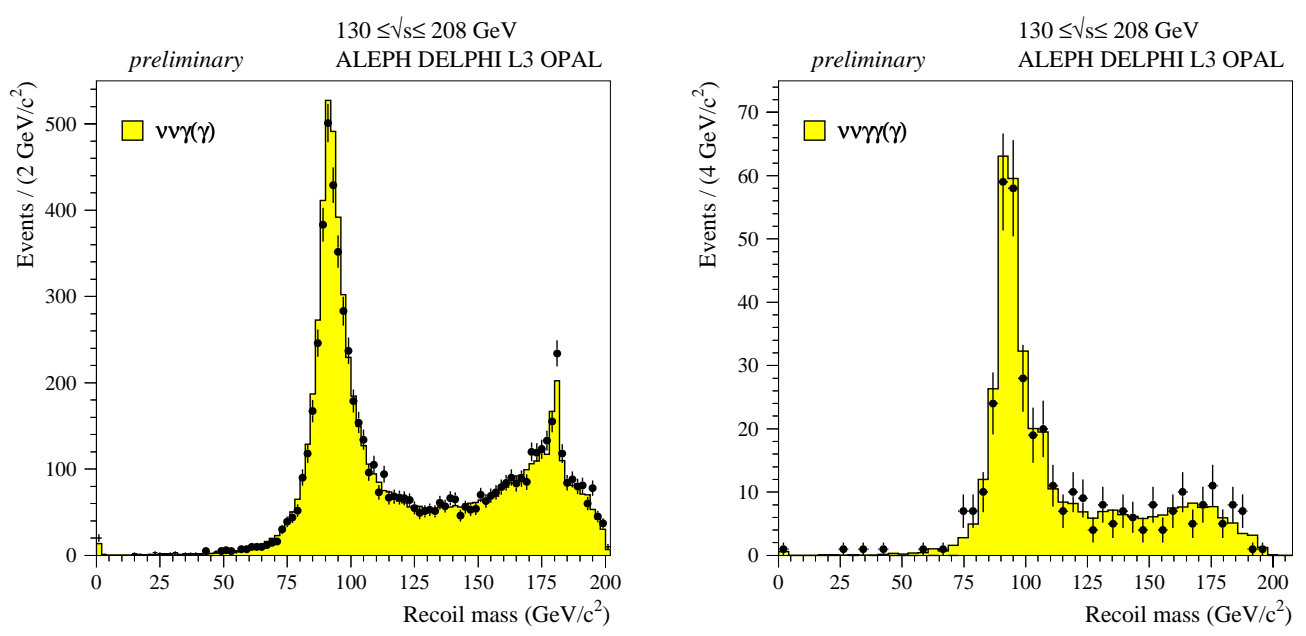

Figure 17: Combined LEP data on isolated single (left) and double (right) photon production compared with the SM expectations

$\mathrm{e}^{+} \mathrm{e}^{-} \rightarrow \chi_{2} \chi_{2}$ with both $\chi_{2} \rightarrow \chi_{1} \gamma[\overline{6} \overline{2} \overline{2}]$, and $\mathrm{e}^{+} \mathrm{e}^{-} \rightarrow \tilde{\mathrm{G}} \tilde{\mathrm{G}} \gamma, \mathrm{e}^{+} \mathrm{e}^{-} \rightarrow \tilde{\mathrm{G}} \chi_{1}$ with $\chi_{1} \rightarrow \tilde{\mathrm{G}} \gamma$ and $\mathrm{e}^{+} \mathrm{e}^{-} \rightarrow \chi_{1} \chi_{1}$ with both $\chi_{1} \rightarrow \tilde{\mathrm{G}} \gamma\left[\overline{6} \overline{3_{1}}\right]$ where $\tilde{\mathrm{G}}$ is a light gravitino in (e.g.) GMSB models. These channels also provide limits completely outside the SUSY framework, e.g. on real and virtual graviton effects possibly made much stronger by graviton propagation

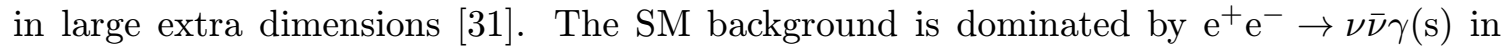
both channels.

On the other hand some SUSY searches require the combined study of many channels. For example limiting the $\tilde{\tau}$ mass in GMSB scenarios in which the gravitino $\tilde{\mathrm{G}}$ is the LSP and $\tilde{\tau}$ the NLSP ('Next to Lightest ..') requires the combination of 3 quite different searches $[\overline{6} \overline{3}]$ because the $\tilde{\mathrm{G}}$ lifetime rises rapidly with its mass. The $\tilde{\chi}^{0}$ mass limit [ $[\overline{6} \overline{5}]$, commonly evaluated in one or more varieties of SUGRA, is perhaps the most complex and arguably the most model-dependent of all.

While LEP searches of course have limited mass reach, it is noteworthy that in their mass range they are often more complete than searches at higher energy hadron machines: e.g. CDF squark searches reach higher masses but, because of the very clean conditions, the LEP squark searches reach much smaller mass differences $\Delta \mathrm{M}$ between the squark and neutralino (supposing $\tilde{q} \rightarrow \tilde{\chi}^{0} q$ ) where the visible quark jets have very low energy [í 62 2]

But finally, no signals of new physics were found. However, the absence of SUSY

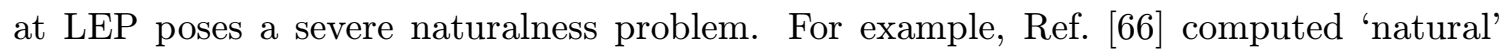
distributions of $\tan \beta$ and various SUSY masses in mSUGRA, see Fig. 1i 1 . Only $\sim 30 \%$ of the points survived the LEP1 data, only $\sim 5 \%$ survived the LEP2 data analysed by ICHEP98, and only $\sim 1 \%$ of the original mSUGRA phase space still survives today, now that the SM Higgs mass limit has risen from 95 to $114 \mathrm{GeV}$. The problem is even more severe in the GMSB and AMSB scenarios (see also below). 

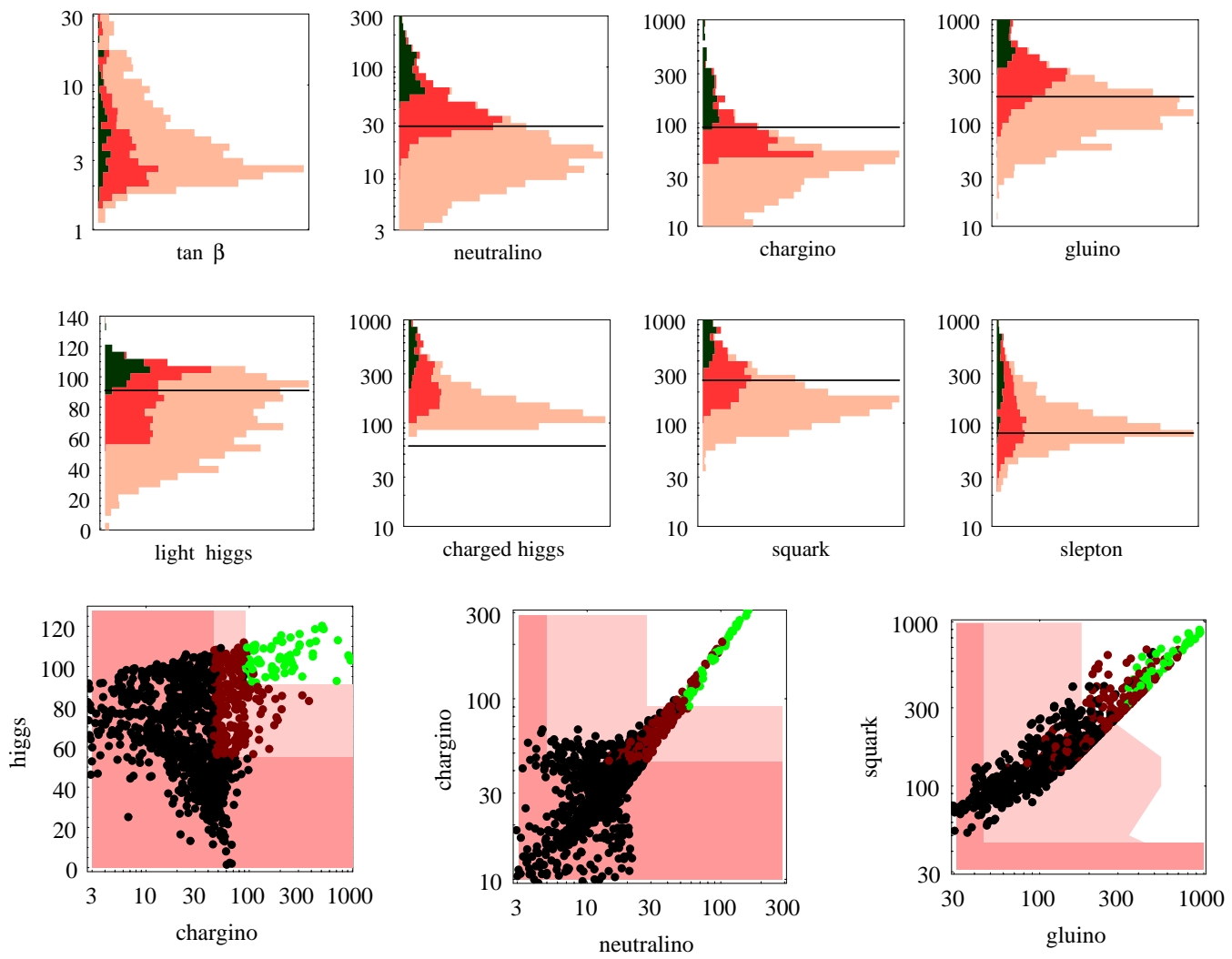

Figure 18: 'Natural' distributions of $\tan \beta$ and various SUSY masses in mSUGRA taken from Ref. $\left[6 \overline{6}^{\prime}\right]$. In the histograms (upper rows), light red shows the a priori natural distribution, dark red the $\sim 30 \%$ surviving LEP1 data, and black the $\sim 5 \%$ surviving LEP2 at ICHEP 98 . In the 2D plots (lower row), the latter are shown in green. Only $\sim 1 \%$ of the original mSUGRA phase space still survives today now that, in particular, the SM Higgs mass limit has risen from 95 to $114 \mathrm{GeV}$, see $2 \mathrm{D}$ plot at bottom left

\section{GLOBAL SUMMARY}

What did LEP achieve?

The new physics initially anticipated $(\mathrm{W}, \mathrm{Z})$ was there. Due to the clean initial situation, hermetic detectors, etc, it was probed with unprecedented precision, typically 2 orders of magnitude better than before LEP started (e.g. $\mathrm{m}_{\mathrm{Z}}$ was measured to $\pm 2.1 \mathrm{MeV}$, $\Gamma_{\mathrm{Z}}$ to $\pm 2.3 \mathrm{MeV}, \mathrm{N}_{\nu}=3$ to 1 part in $350, \mathrm{R}_{\mathrm{b}}$ to $\pm 0.3 \%$ which is 20 times better than initial hopes, $\mathrm{m}_{\mathrm{W}}$ to $\pm 39 \mathrm{MeV}$ and still improving); and $\mathrm{m}_{\text {top }}$ was predicted correctly, universality was tested at the $\sim 1$ per mille level in EW interactions and to $1 \%$ in QCD, the cancellation of WW production amplitudes required by gauge theory was tested at the $1 \%$ level, and purely weak loop corrections at the $\sim 10 \%$ level. LEP also brought deeper knowledge of heavy flavours, deeper understanding of QCD, and showed that GUTs work with SUSY but not without. And the new particle searches were remarkably complete and rigorous, leaving very few corners still unexplored (and squeezing mSUSY into a very tight 
one!). But there were no further surprises. Apparently nature chose to be at her most boring?
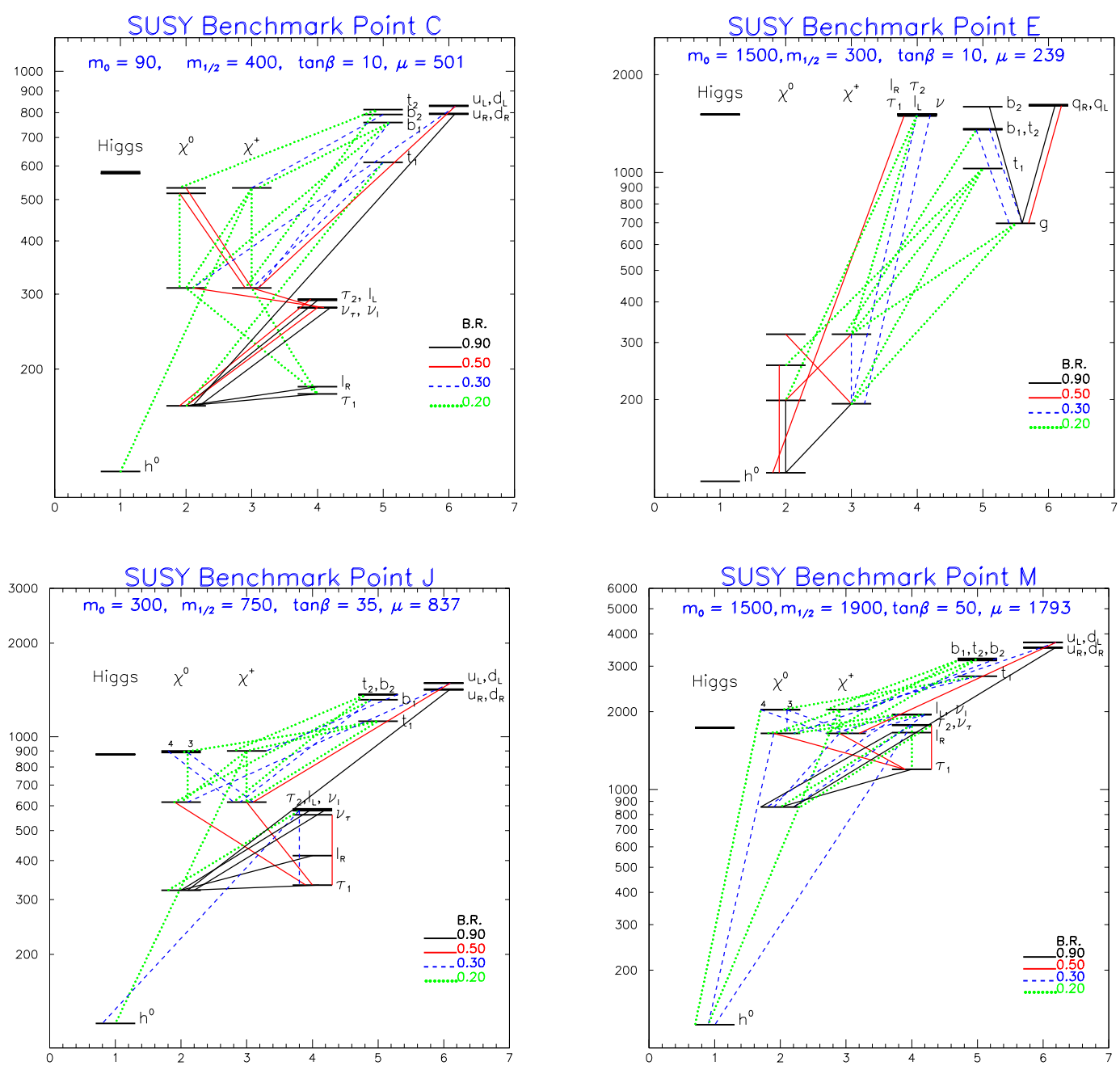

Figure 19: Higgs and SUSY particle mass spectra (vertical scale in GeV) for 4 of 13 benchmark scenarios proposed in Ref. [60 SUSY masses may all be very high (e.g. see bottom right) but one Higgs always lies below $124 \mathrm{GeV}$

But perhaps this was the biggest surprise of all! Although pushed beyond all reasonable limits "the dog did not bark" (pace Sherlock Holmes). In "Silver Blaze", Inspector Gregory asked Sherlock Holmes 'To what would you wish to draw my attention?', and Holmes answered: 'To the curious incident of the dog in the night-time'. To the objection that 'The dog did nothing in the night-time', Holmes replied: 'THAT was the curious incident!'.

So we learned that Glashow's "least probable" scenario was $100 \%$ correct! Here are a few opinions (some slightly paraphrased) on what this meant.

Frank Wilczek (CERN LEPfest, November 2000): "The historic achievement of LEP has been to establish with an astonishing degree of rigor and beyond all reasonable doubt 


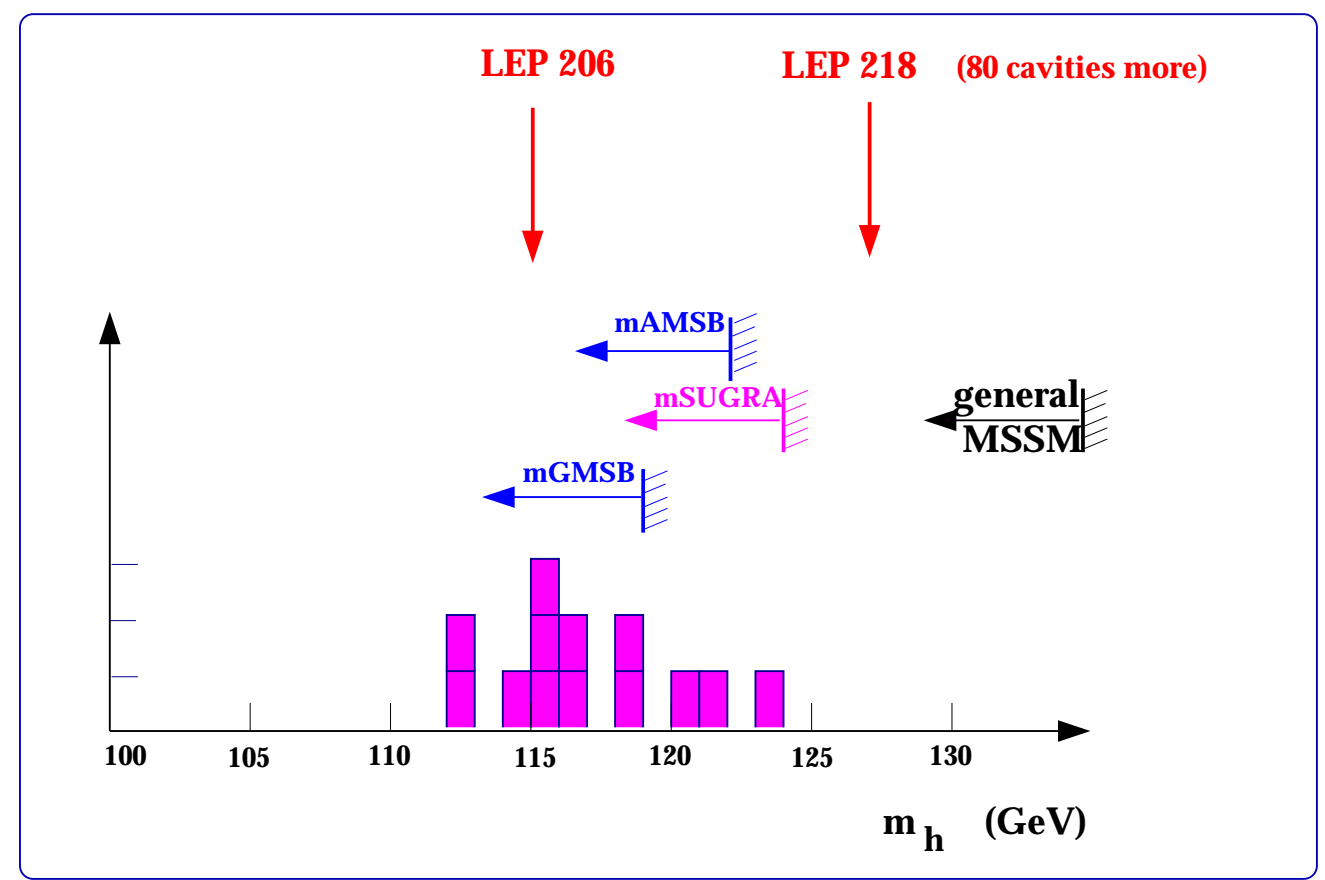

Figure 20: Higgs mass values for the 13 benchmark scenarios proposed in Ref. [60 post-LEP range of constrained mSUGRA possibilities compared with the Higgs mass reach actually achieved and the reach that could have been achieved if it had not been decided to discontinue superconducting rf cavity production in 1996

what will stand for the foreseeable future - perhaps for all time - as the working Theory of Matter ..... and to give us some very definite and specific clues for what lies beyond".

Chris Llewellyn-Smith (UK LEPfest, June 2001): "We made big conceptual progress: relativistic quantum field theory IS the right language, all forces are due to local symmetries (gauge fields), nature hides symmetries (chiral symmetry, EW symmetry, SUSY?), and ElectroWeak theory is a real Quantum Field Theory".

John Ellis (CERN Academic Training, June 2001): "The Standard Model is no longer the Standard Model, it is the Standard Theory".

But part of "the dog did not bark" surprise is that, after searching NEARLY the whole phase space (even if in an admittedly VERY constrained model), SUSY wasn't there! Very surprising! But we could have done better .....

With hindsight, LEP clearly should have had more superconducting rf. Recently Ref. [î́0] proposed 13 benchmark points to span the post-LEP range of constrained mSUGRA possibilities. In all cases the Higgs was SM-like with a mass below $124 \mathrm{GeV}$ (see Fig. II $\underline{g}^{\top}$ ), while Ref. [6] found $\mathrm{m}_{\mathrm{H}} \mathrm{HAD}$ to be below 124 (mSUGRA), 119 (mGMSB) and 122 (mAMSB) $\mathrm{GeV}$ with uncertainties of $\sim \pm 5 \mathrm{GeV}$ due to the error on $\mathrm{m}_{\text {top }}$. As Fig. $2 \overline{0}_{1}^{\prime}$ shows, all of these cases would have been in LEP2's range, with either the Higgs being discovered by now or all currently popular MSSM models being essentially excluded, if it had not been decided to discontinue superconducting rf cavity production in 1996. 


\section{CONCLUDING REMARKS}

The LEP Working Groups, in which all 4 LEP expts worked together for a common aim, whether combining results or working with theorists or LEP machine experts, were a great success. They engendered LEP's great spirit of competition AND cooperation between the expts, but were probably a direct result of the data being split as many as 4 ways. It would be nice to think that the same spirit could transfer to the LHC expts.

All of the LEP experimenters owe very big 'THANK-YOU's both to all the theorists and phenomenologists for computing the EW radiative corrections so precisely and for all the event generators and other tools like Zfitter, and to all the LEP machine people for their superb achievements over many years and especially for their enormous enthusiasm and effectiveness in last year's Higgs hunt, which were an inspiration to the experimenters! Without the help of all of them, the experimenters could not have produced the physics.

But LEP analysis is not finished yet, and the papers still to come are the FINAL papers which must do justice to two decades of hard work by very many people.

\section{Acknowledgments}

Many thanks to Daniel Treille, Guy Wilkinson and all the LEP Working Groups for permission to use the beautiful summary plots they had prepared, and to all my LEP colleagues for all their help with this talk and over the years.

\section{References}

[1] B.Richter, Very high energy electron-positron colliding beams for the study of the weak interaction, CERN/ISR-LTD/76-9, March 1976, reprinted as Appendix 1 of ref. [in]].

[2] L.Camilleri et al., Physics with very high-energy $e^{+} e^{-}$colliding beams, Yellow Report CERN-76-18, Nov 1976.

[3] CERN/ISR-LEP 78-17, known as the 'Blue book'.

[4] LEP Summer Study, Les Houches and Geneva, Yellow Report CERN-79-01, 2 vols, S.L.Glashow p.285, J.B.Adams p.281.

[5] Proc. Int. Conf. on HEP, Munich 1988, D.H.Perkins, p.446.

[6] Proc. Int. Symp. on Lepton and Photon Intns., Stanford 1989, F.Sciulli, p.452.

[7] See plenary review talk by Dave Charlton, these Proceedings.

[8] Proc. Int. Symp. on Lepton and Photon Intns., Stanford 1989, G.Feldman, p.234.

[9] Proc. XXVth Rencontre de Moriond, ' $\mathrm{Z}^{0}$ Physics', L.Rolandi, p.247.

[10] S.Sarkar, 'Rept. Prog. Phys. 59 (1996) 14931, hep-ph/9602260'.

[11] H.Burkhardt and B.Pietrzyk, LAPP-EXP-2001-03, Feb 2001, subm. to Phys. Lett. B.

[12] See talk by J.Chen, these Proceedings.

[13] A.Martin et al., 'Phys. Lett. B 
[14] Proc. Int. Euro. Conf. on HEP, Marseille 1993, J.Lefrancois, p.671.

[15] Proc. Int. Conf. on HEP, Glasgow 1994, Vol 2, D.Schaile p.27.

[16] Proc. Int. Conf. on HEP, Glasgow 1994, Vol 2, H.B.Jensen p.3, P.D.Grannis p.15.

[17] Proc. Int. Euro. Conf. on HEP, Brussels 1995, parallel session contributions summarised (plenary review) by A.Menzione p.933.

[18] Proc. Int. Conf. on HEP, Dallas 1992, C.Moisan p.670.

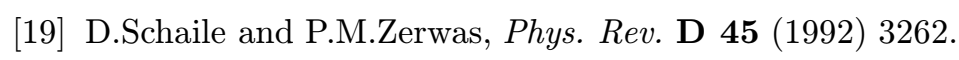

[20] Proc. Int. Euro. Conf. on HEP, Brussels 1995, see for example H.E.Haber, p.477.

[21] Proc. Int. Euro. Conf. on HEP, Brussels 1995, D.Charlton, p.266

[22] F. Caravaglios, Proc. Int. Euro. Conf. on HEP, Brussels 1995, p.57;

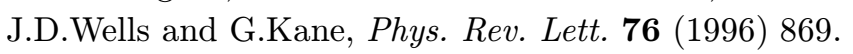

[23] See talk by S.Mele, these Proceedings.

[24] See for example the talks by M.Paganoni, P.Casado, H.Ruiz, P.Hansen and V.Serbo, these Proceedings.

[25] See talks by H.Ruiz, O.Pooth and D.Duchesneau, these Proceedings.

[26] See talk by P.Hansen, these Proceedings.

[27] See talk by V.Serbo, these Proceedings.

[28] See for example F.Caravaglios and G.G.Ross, 'P hyss. Lett. B $\mathbf{3} 4 \mathbf{6}-(\overline{1} 9 \overline{9})$ 159';

J.H.Field, Mod. Phys. Lett. A 13 (1998) 1937, also as thep-ph/9801355;

J.H.Field and D.Sciarrino, Mod.Phys.Lett. A 15 (2000) 761: also as hep-ex/9907018;

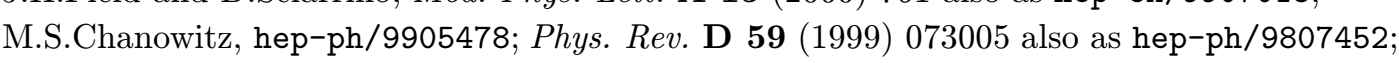
and hep-ph/01040245

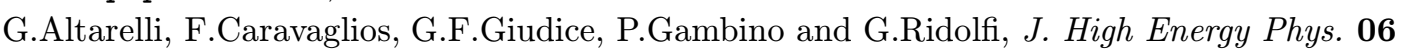

(2001) 018 also as hep-ph/0106029!

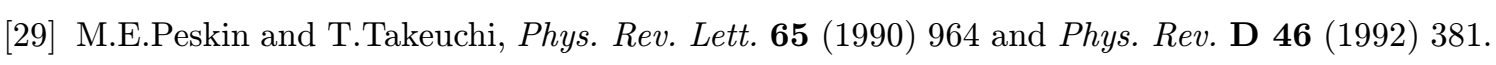

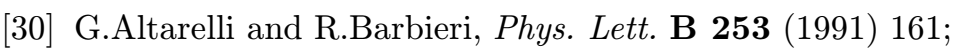

G.Altarelli, R.Barbieri and S.Jadach, 'Nucl. Phys. $\mathbf{B} \mathbf{3} \mathbf{9}(\overline{1} \overline{9} 92)$

G.Altarelli, R.Barbieri and F.Caravaglios, Nucl. Phys.

[31] See talks by J.Holt and S.Shevchenko, these Proceedings.

[32] See for example the talk by M.Roth, these Proceedings.

[33] See talk by R.Chierici, these Proceedings.

[34] See talks by H.Rick, S.Villa, A.Oh and M.Biglietti, these Proceedings.

[35] See talk by K.Osterberg, these Proceedings.

[36] See talk by F.Palla, these Proceedings.

[37] See talk by D.Miller, these Proceedings.

[38] See plenary review talk by Z.Ligeti, these Proceedings.

[39] See talks by C.Weiser and J.Thom, these Proceedings. 
[40] See talk by P.Casado, these Proceedings.

[41] G.Altarelli, 'Ánn. Rev. Nucl. Part. Sci. 39 (1989) $35 \overline{1}$.

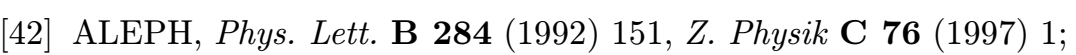

DELPHI, Phys. Lett. B 255 (1992) 151, Phys. Lett. B 414 (1997) 401;

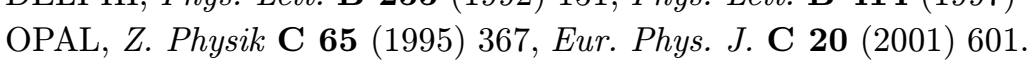

[43] See talk by D.Wicke, these Proceedings.

[44] See talk by P.Bambade, these Proceedings.

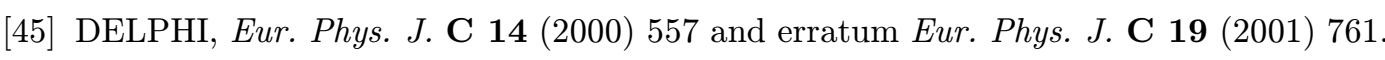

[46] Reference [45i] and references therein.

[47] See talks by J.Vossebeld and D.Muller, these Proceedings.

[48] See talks by T.Aziz, G.Alexander, O.Pooth and D.Duchesneau, these Proceedings.

[49] See talks by A.Finch, L.Malgeri, M.Przybycien and V.DelDuca, these Proceedings.

[50] See plenary review talk by F.Gianotti, these Proceedings.

[51] Proc. XXVth Rencontre de Moriond, ' $\mathrm{Z}^{0}$ Physics', D.Lellouch p.127, G.Wormser p.137, M.Kasemann p.165, C.Dionisi p.183.

[52] Proc. 25th Int. Conf. on HEP, Singapore 1990, F.Dydak p.3.

[53] See talks by D.Zer-Zion and I.Josa, these Proceedings.

[54] ECFA Workshop on LEP200, Aachen 1986, CERN-87-08 and ECFA-87-108, D.H.Perkins p.1, S.L.Wu p.312, see also Ref. 榢.

[55] A.L.Read in Yellow Report CERN-2000-005, p.81 (2000).

[56] See talks by J.Marco and F.Gianotti, these Proceedings.

[57] The term 'exotic' now commonly refers to all new physics searches other than for the SM or MSSM Higgs or for SUSY. See talks by J.Holt, S.Shevchenko, M.Pimenta, D.Zer-Zion and I.Josa, these Proceedings.

[58] U.Amaldi, W.deBoer and H.Furstenau, 'P

U.Amaldi, W.deBoer, P.H.Frampton and H.Furstenau, Phys. Lett. B $281-(1992) 374$.

[59] J.H.Schwarz, "Introduction to Superstring theory", hep-ex/0008017.

[60] M.Battaglia, A.DeRoeck, J.Ellis, F.Gianotti, K.T.Matchev, K.A.Olive, L.Pape and G.Wilson, "Proposed Post-LEP Benchmarks for Supersymmetry", hep-ph/0106204!

[61] B.Richter, LP99 summary talk, http://lp99.slac.stanford.edu/db/program.asp.

[62] See talk by B.Clerbeau, these Proceedings.

[63] See talk by C.Rembser, these Proceedings.

[64] See talks by M.C.EspiritoSanto and J.Holt, these Proceedings.

[65] See talk by P.Azzuri, these Proceedings.

[66] L.Giusti, A.Romanino and A.Strumia, Nucl. Phys. B 550 (1999) 3inep-ph/9811386.

[67] S.Ambrosanio, A.Dedes, S.Heinemeyer, S.Su and G.Weiglein, hep-ph/0106255i. 